Research paper

\title{
ORACLE: A mission concept to study Mars' climate, surface and interior
}

\author{
Antonio Genova \\ Sapienza, University of Rome, Department of Mechanical and Aerospace Engineering, Italy
}

\section{A R T I C L E I N F O}

\section{Keywords:}

Mars mission

Radio science

Altimetry

Mars seasonal polar deposits

Mars interior

\begin{abstract}
A B S T R A C T
A comprehensive knowledge of the Martian climate requires a better understanding of the formation and evolution of the polar caps. Accurate measurements of mass, volume, and composition of the seasonal polar deposits would enable a precise characterization of the surface-atmosphere interactions relevant to the $\mathrm{CO}_{2}$ cycle. The acquisition of these science data is only possible through a dedicated orbital mission at Mars. ORACLE (Orbital Radio science and Altimetry for CLimate Experiment) is a mission concept that enables the monitoring of the $\mathrm{CO}_{2}$ mass exchange between the polar caps and the atmosphere with a pair of spacecraft in a $250 \times 320 \mathrm{~km}$ orbit about Mars. A preliminary design of the mission configuration is presented in this study including the scientific payload that consists of a radio science system for inter-satellite tracking, a laser altimeter, and an infra-red camera. These instruments also provide data that are fundamental to investigate Mars' internal structure and atmosphere. ORACLE is, therefore, a multi-disciplinary mission that is conceived to fulfill the constraints of a medium-class mission.
\end{abstract}

\section{Introduction}

The exploration of the planet Mars has always represented one of the main objectives of international space programs. Several missions have been designed to enhance our understanding of the planet's environment, especially, regarding the existence of present or past habitable conditions. In the last two decades, the data collected by rovers, landers, and orbiters have enabled to expand our knowledge of Mars from its deep interior to the upper atmosphere. The ESA-Roscosmos mission to Mars ExoMars - Trace Gas Orbiter (TGO) [1] and the NASA Mars Atmosphere and Volatile EvolutioN (MAVEN) [2] are currently orbiting Mars to investigate its habitability by providing the most detailed inventory of Martian atmospheric gases and by determining the mechanisms that led Mars to lose its atmosphere, respectively. The NASA Interior Exploration using Seismic Investigations, Geodesy and Heat Transport (InSight) mission [3] has successfully landed on Mars on 26 November 2018 to measure its seismic activity, internal heat flow, and rotational state that will provide crucial information on the planet's interior. Previous orbital missions (e.g, Mars Express, Mars Global Surveyor - MGS, Mars Odyssey, and Mars Reconnaissance Orbiter MRO) have also investigated the Martian climate, weather, atmosphere, and geology yielding outstanding findings. However, fundamental scientific questions on Mars' climate and interior are still open. The mission concept, named Orbital Radio science and Altimetry for CLimate Experiment (ORACLE), is focused on the fulfillment of these key science objectives.
Mars ORACLE is, therefore, an interdisciplinary mission that is dedicated to a comprehensive investigation of the Martian climate, surface, and internal structure. One of the main scientific goal is the characterization of the seasonal polar deposits by measuring their mass from gravity and volume from the combination of altimetry and imaging data. The quantities of mass exchange between the polar caps and the atmosphere are still highly uncertain, and the local $\mathrm{CO}_{2}$ is mainly based on theoretical predictions of General Circulation Models (GCM) of the Martian atmosphere [4] and on sparse altimetry [5] and neutron spectroscopy data [6]. ORACLE is well-suited to provide measurements for an in-depth understanding of the atmospheric and ice science on Mars.

The mission design was studied to accomplish these challenging scientific objectives by following M. Wolff concept to measure planetary gravity field using a pair of identical satellites [7] that paved the way for the successful NASA missions Gravity Recovery and Climate Experiment (GRACE) [8], and Gravity Recovery and Interior Laboratory (GRAIL) [9]. Therefore, Mars ORACLE configuration consists of a pair of spacecraft in the same near-circular near-polar orbit at $\sim 250 \times 320 \mathrm{~km}$ altitude and inter-spacecraft distance of $\sim 300 \mathrm{~km}$. The science instrumentation onboard ORACLE are a radio science (RS) system for tracking from Earth and between the probes, an altimeter (e.g. laser), and a wide-angle camera (e.g. InfraRed, IR). These three instruments are designed to provide a comprehensive dataset that enables the determination of time-varying gravity and topography of Mars, and the monitoring of the extent of the seasonal polar deposits.

E-mail address: antonio.genova@uniroma1.it. 
The simultaneous estimates of $\mathrm{CO}_{2}$ mass, volume, and composition lead to unique observations of the densities of the seasonal polar deposits.

The measurements collected by the three scientific instruments allow addressing other fundamental questions related to atmospheric sciences, geodesy, and geophysics. Inter-satellite tracking may provide measurements of the content of charged particles in the Martian ionosphere, and the laser altimeter may also detect clouds at altitudes lower than $10 \mathrm{~km} \mathrm{[10].} \mathrm{Furthermore,} \mathrm{radio} \mathrm{and} \mathrm{altimetry} \mathrm{data} \mathrm{enable} \mathrm{the}$ retrieval of high-resolution gravity and topographic maps that are pivotal to determine the processes that led to the formation of specific geological features including the permanent polar layered deposits and the history of the Martian volcanoes. The ORACLE dataset also helps to characterize the core and mantle of Mars by measuring gravitational tides, which provide complementary information to the Insight mission.

This paper presents in detail the Mars ORACLE mission concept, and it is structured as follows. In Section 2, we describe the scientific goals of the mission. In Section 3, we show in detail the mission profile, and the flight system including the scientific payload. In Section 4, we present the numerical simulations of the gravity and altimetry investigations including a description of orbital error sources. In Section 5 , we discuss the conclusions of this study.

\section{Scientific goals}

The ORACLE mission to Mars aims to accomplish outstanding science goals regarding the habitability of the solar system, in relation to the evolution of the different Mars' systems, from the deep interior to the atmosphere, and their interactions. Table 1 shows the major fundamental scientific questions and objectives of the ORACLE mission. In Sections 2.1 and 2.2, we describe in detail the contribution that ORACLE would provide to the atmospheric sciences, geodesy and geophysics by using a dedicated RS instrumentation for inter-satellite tracking, a laser altimeter and an IR camera (Section 3.3).

\subsection{Martian climate}

The climate of Mars is mainly controlled by the mass exchange between the surface and atmospheric $\mathrm{CO}_{2}$ reservoirs [11]. GCMs provide variations in the atmospheric constituents, which are based on theoretical models that are adjusted to match measured pressure profiles [12-14]. These predicted observations enable an indirect retrieval of the global amount of $\mathrm{CO}_{2}$ that accumulates and sublimates in the polar regions. Neutron spectroscopy [15] and gravity experiments [16] of orbital missions around Mars have yielded independent measurements of the seasonal $\mathrm{CO}_{2}$ ice mass evolution. Other studies also looked at the tiny effects on Mars' orientation due to the atmosphere-polar caps interactions $[17,18]$, which do not provide significant information on the entire $\mathrm{CO}_{2}$ cycle. Furthermore, GCMs are still not accurate enough because of the sparse dataset of pressure profiles on the Martian surface, and experimental estimates are strongly limited by the poor resolution of the time-varying gravity field $[19,20]$ and of the neutron flux data [21]. Therefore, the knowledge of the mass and energy exchange between the atmosphere and the polar caps is still uncertain.

To address this fundamental objective, the Mars ORACLE mission is capable of measuring the gravity anomalies induced by the seasonal $\mathrm{CO}_{2}$ deposits. An accumulation of $1 \mathrm{~m}$-thick $\mathrm{CO}_{2}$ frost results in gravity anomalies of the order of $\sim 0.04 \mathrm{mGal}$, which is computed by assuming a simplified Gauss' law for gravity $\sim 2 \pi G \rho h$, where $G$ is the gravitational constant, $\rho \sim 900 \mathrm{~kg} \mathrm{~m}^{-3}$ and $\mathrm{h}=1 \mathrm{~m}$ for $\mathrm{CO}_{2}$ ice density and thickness, respectively. Previous gravity investigations based on MGS, Mars Odyssey and MRO orbit determination with Deep Space Network (DSN) radio tracking data only enabled the estimation of an average global distribution of $\mathrm{CO}_{2}$ in both polar caps with a spatial resolution of $\sim 2500-3000 \mathrm{~km}$ and accuracies of $\sim 0.05 \mathrm{mGal}[19,20]$. Fig. 1 shows the measured gravity signal induced by $\mathrm{CO}_{2}$ condensation in the northern hemisphere during its winter solstice (Martian solar longitude $L_{s}$ 

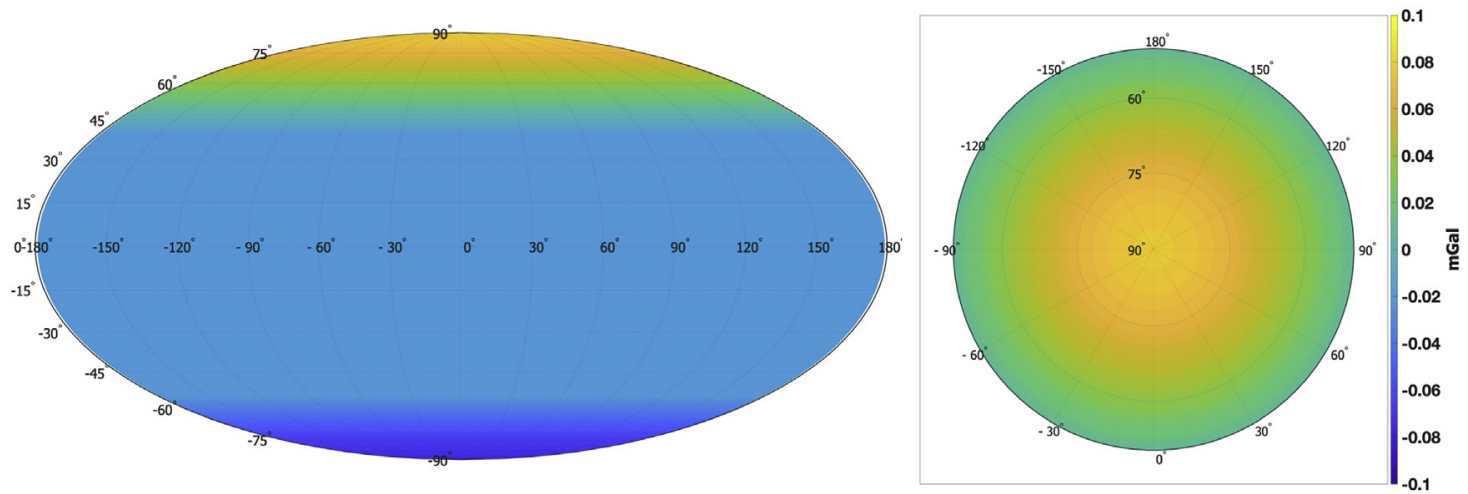

Fig. 1. Maps of gravity anomalies (mGal) induced by the condensation and sublimation of $\mathrm{CO}_{2}$ in a Mollweide projection for the entire planet (left) and in a polar stereographic projection for the northern hemisphere (right). These maps are computed at the winter solstice in the northern hemisphere. These anomalies are based on the time-varying gravity solution $\left(J_{2}(t)\right.$ and $\left.J_{3}(t)\right)$ retrieved by Genova et al. [20].

$=270^{\circ}$ ). The radio science investigation of the Mars ORACLE mission is focused on the accurate determination of mass variations of $\mathrm{CO}_{2}$ ice at spatial scales of $\sim 360 \mathrm{~km}$ with accuracies of $\sim 0.01 \mathrm{mGal}$ (i.e., $\sim 30 \mathrm{~cm}$ of $\mathrm{CO}_{2}$ frost).

Precise measurements of short-wavelength gravity anomalies are based on a dual-spacecraft configuration with inter-satellite radio tracking data similarly to the NASA missions GRACE for the Earth [8] and GRAIL for the Moon [9]. The attainable accuracies of ORACLE's geodetic observations rely strongly on the RS instrumentation onboard the spacecraft and the mapping orbit selected to monitor the timevarying gravitational effects. The RS system onboard the ORACLE spacecraft differs significantly from the NASA missions GRACE and GRAIL designs (Section 3.3), but it guarantees highly accurate intersatellite radio tracking data to measure precise seasonal mass variations. To enable a uniform coverage of the Martian surface with radio tracking and laser altimeter data, the orbit of the two spacecraft is selected to be similar to the current orbit of the MRO spacecraft $(\sim 250 \times 320 \mathrm{~km})$ by assuming an inter-satellite distance of $300 \mathrm{~km}$ (Section 4).

The scientific payload of the ORACLE mission includes a wide-angle Thermal-InfraRed (TIR) camera to monitor the physical extent of the polar caps. The definition of the thermal cap edges helps constrain the region where $\mathrm{CO}_{2}$ frost accumulates leading to a detailed mapping of its inter-annual evolution [22]. The laser altimeter onboard one of the two spacecraft provides accurate measurements of the thickness of the seasonal deposits that combined with the physical extension seen by the camera enable a detailed mapping of the accumulated frost volume.

The simultaneous estimation of the mass, volume, and composition of the seasonal polar deposits is fundamental for the characterization of their density and porosity at high spatial resolution during an entire Martian year.

\subsection{Mars' interior and surface}

The Mars ORACLE mission is also focused on fundamental scientific objectives related to geodesy and geophysics. The scientific instrumentation onboard the two spacecraft enable highly accurate measurements of the static gravitational and topographic fields to address science questions regarding the interior structure and surface of Mars. The most accurate global map of Mars' topography, which has a spatial resolution of $\sim 1 \times 2 \mathrm{~km}^{2}$ at the equator [23], is still based on the Mars Orbiter Laser Altimeter (MOLA) data collected by the NASA mission MGS [24,25]. The gravity field of Mars was recently estimated by combining the radio tracking data of the NASA missions MGS, Mars Odyssey, and MRO leading to a gravity anomaly map with a spatial resolution of $\sim 110-125 \mathrm{~km}$ in the southern and northern hemispheres, respectively $[20,26]$. The determination of the crustal thickness by comparing gravity and topography is limited by the poorer resolution of the gravitational anomaly map. The combination of gravity and topography is, therefore, important to enhance our knowledge of Mars' outer layers density and thickness.

High-resolution maps of Mars' gravity and topography represent one of the main objectives of the ORACLE mission (Section 4). These resulting geophysical measurements are fully adequate to answer fundamental questions regarding the crust of Mars. Similar to the GRAIL mission for the Moon [27], highly accurate gravity and topography data enable the determination of the bulk density and porosity of the crust [28], and provide finer details of the global crustal thickness map. An enhanced resolution of the gravity anomalies over the polar caps, for example, is important to determine the bulk densities of the permanent polar deposits (and hence composition) and the thickness of the elastic lithosphere, which is related to the local heat flow of Mars [29]. The present-day heat flow at the polar caps plays an important role in determining whether basal melting ever occurred beneath the polar caps, and this heat flow could supplement the value obtained by the InSight [3] mission that landed on Mars at equatorial latitudes. To accomplish this science goal, the laser altimeter aboard ORACLE is also dedicated to observe topographic relief with vertical accuracies of $<30 \mathrm{~cm}$ (Section 3.3), which are well-suited to enhance MOLA topography locally and globally. These improvements are necessary to elucidate how specific geological features formed, and, in particular, to have a better understanding on the layering that is observed in the polar caps.

The gravity investigation of the ORACLE mission also provides crucial information on Mars' deep interior. The gravitational quadrupole term (i.e., $J_{2}$ ) and tides (i.e., Love numbers $k_{2}$ and $k_{3}$ ) represent essential quantities to determine the degree of internal differentiation and to constrain physical properties of the core and mantle. Furthermore, a precise estimate of the phase lag of the Love number $k_{2}$ helps the characterization of the rheology of the internal layers. These geodetic measurements of the ORACLE mission in combination with a highly accurate knowledge of Martian precession and nutation, which will be determined by the NASA mission InSight and the Lander Radio science (LaRa) experiment [30,31] on ExoMars 2020 [1], will enable to infer the size and composition of the core and to constrain the rheological properties of the mantle.

\section{Mission configuration}

The Mars ORACLE mission concept is conceived to be compliant with ongoing space mission programs of ESA (e.g., M-class) and NASA (e.g., Discovery). In Section 3.1, we describe the mission profile including the Earth-Mars transfer module and the mapping orbit. The flight system of the mapping phase consists of a pair of small spacecraft (Section 3.2) orbiting Mars at low altitudes with three scientific 
Table 2

Mass and power of the ORACLE Transfer Module (OTM), and ORACLE-A and -B spacecraft.

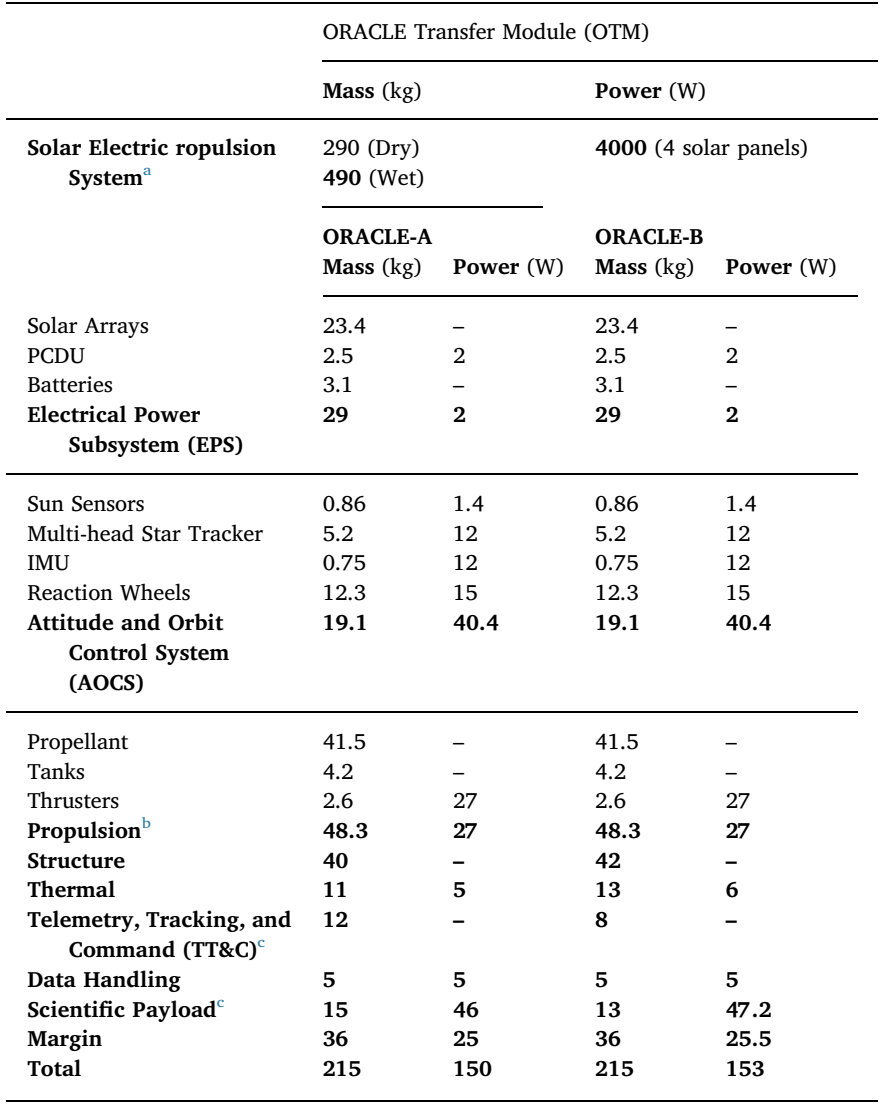

a A pair of thrusters with a maximum thrust of $75 \mathrm{mN}$.

b The propulsion system of the spacecraft includes two sets of 4 hydrazine thrusters.

c The scientific payload mass and power demand account for part of the TT\& C elements (Fig. 3 and Table 3 ).

payloads onboard (Section 3.3).

\subsection{Mission profile}

By assuming that a four-stage launch vehicle provides a sufficient $\Delta-V$ to a total wet mass of $\sim 1000 \mathrm{~kg}$ to escape Earth's gravity, the small orbiters are integrated into a transfer module that hosts a solar electric propulsion system. This additional module enables a low-thrust relative orbital transfer from Earth to Mars [32].

The solar electric propulsion system is based on the technology of BepiColombo's Mercury Transfer Module (MTM) [33] with significant reduced size and mass. The ORACLE Transfer Module (OTM) relies on the command and control system of one of the two spacecraft, similarly to BepiColombo's MTM that is supported by the Mercury Planetary Orbiter (MPO). Table 2 shows the OTM mass and power consumption by assuming four solar panels ( $\sim 4 \mathrm{~m}^{2}$ area each) and two thrusters (one in cold redundancy) with a maximum thrust of $75 \mathrm{mN}$ and a specific impulse $\left(I_{s p}\right)$ of $\sim 3000-4000 \mathrm{~s}$ (e.g., T6 ion engine [34]). The OTM operates during the cruise trajectory to reach a Mars Highly Elliptical Orbit (e.g., $\sim 500 \times 96,000 \mathrm{~km})$ in $\sim 2-3.5$ years with a delivered mass of $\sim 800 \mathrm{~kg}$ [35]. The OTM is jettisoned at the apoapsis altitude of the eccentric orbit with similar operations foreseen for the MTM of the BepiColombo mission [33] to satisfy planetary protection requirements.

The two spacecraft then reach the target orbit for the primary science phase (PSP) after few months with a combination of orbit correction maneuvers (OCMs) and aerobraking operations to reduce significantly the apoapsis altitude leading to a near-circular polar orbit. This approach is high-risk since the maneuvers are used to control the aerobraking corridor and to avoid collision with other spacecraft. The main advantage of aerobraking, however, is a substantial propellant saving, as demonstrated by the NASA mission Mars Odyssey and MRO, which saved in excess of $\sim 300 \mathrm{~kg}$ and $\sim 580 \mathrm{~kg}$ of propellant, respectively [36]. ESA adopted recently aerobraking for the TGO spacecraft with 950 orbits to change the orbit from $\sim 200 \times 98,000 \mathrm{~km}$ to $\sim 200 \times$ $1050 \mathrm{~km}$. The final TGO orbit was reached after a 16-min propulsive maneuver.

The PSP orbit configuration was determined as a trade-off between orbital and science constraints. The gravity investigation requires, for example, low altitudes to increase the gravity signal in the radio tracking data. However, orbits with altitudes lower than $200 \mathrm{~km}$ are significantly perturbed by the atmospheric drag. Strong drag perturbations on the spacecraft affect severely the inter-satellite tracking leading to a degraded quality of the data. Furthermore, more frequent OCMs are required to maintain the desired orbit. A semi-major axis $a$ of $\sim 3660 \mathrm{~km}$ is, therefore, an adequate compromise for the ORACLE mission profile. The nominal orbit for the PSP is near-circular $(\sim 250 \times 320 \mathrm{~km})$ near-polar $\left(i \sim 93^{\circ}\right)$ with the pericenter located in the southern hemisphere $\left(\omega_{p}=270^{\circ}\right)$, which is approximately the primary science orbit of the NASA mission MRO [37]. This PSP orbit configuration is adopted in the numerical simulations presented in Section 4. This mission scenario represents a conservative case since orbits at lower altitudes $(a \sim 3640 \mathrm{~km}$ ) are feasible. Science data collected at lower altitudes would enhance the scientific return of the mission. The orbital eccentricity and argument of pericenter yield a frozen orbit since the natural drifting due to Mars' gravity zonal harmonics (e.g., $J_{2}, J_{3}, J_{4}$ ) is minimized leading to less frequent orbital correction maneuvers.

To determine the optimal inter-satellite distance between the two spacecraft, we simulated the gravity signal in the spacecraft-to-spacecraft tracking (SST) data induced by a mascon of $0.01 \mathrm{mGal}$ at $55^{\circ} \mathrm{N}$ with an area of $360 \times 360 \mathrm{~km}^{2}$. The magnitude of this mascon corresponds roughly to the gravity anomaly generated by a $\mathrm{CO}_{2}$ ice slab with a thickness of $\sim 30 \mathrm{~cm}$. Fig. 2 shows the gravity signal measured in the Doppler data at different altitudes and inter-spacecraft distances. These measurements were determined as data residuals that are the differences between the simulated observables, which were computed by including the mascon, and the computed observables that were retrieved by using the same dynamical model of the simulated observables except for the mascon. Fig. 2-a is the result of several numerical simulations that sample the dimensional spaces of spacecraft altitude (200-390 km) and relative distance (5-305 km) with a step size of $5 \mathrm{~km}$. These solutions are in agreement with previous studies [38], which demonstrated that large spacecraft separations enhance the recovery of the time-variable gravity signal. A mean altitude of $\sim 285 \mathrm{~km}$ and an inter-spacecraft distance of $300 \mathrm{~km}$ enable a gravity signal of 0.6 $\mu \mathrm{m} \mathrm{s}^{-1}$ that is consistent with the data accuracy of the SST of the ORACLE mission (Section 3.3).

\subsection{Flight system}

The design of the two spacecraft is strictly based on the subsystems that ensure the successful completion of the science operations during the PSP. Miniaturization and integration of each spacecraft subsystem is required to fulfill stringent mission constraints. The orbital insertion, for example, significantly limits the available spacecraft mass and power in orbit about Mars. By accounting for these boundary conditions, we present a system-level view of the two spacecraft, named ORACLE-A and -B (Table 2).

The radio science investigation provides additional constraints on the size and mass of the two spacecraft. To measure accurately the gravity anomalies with SST data, we need to minimize the perturbative effects due to other forces. These dynamical perturbations do not affect significantly the quality of the SST data, which measure the relative 

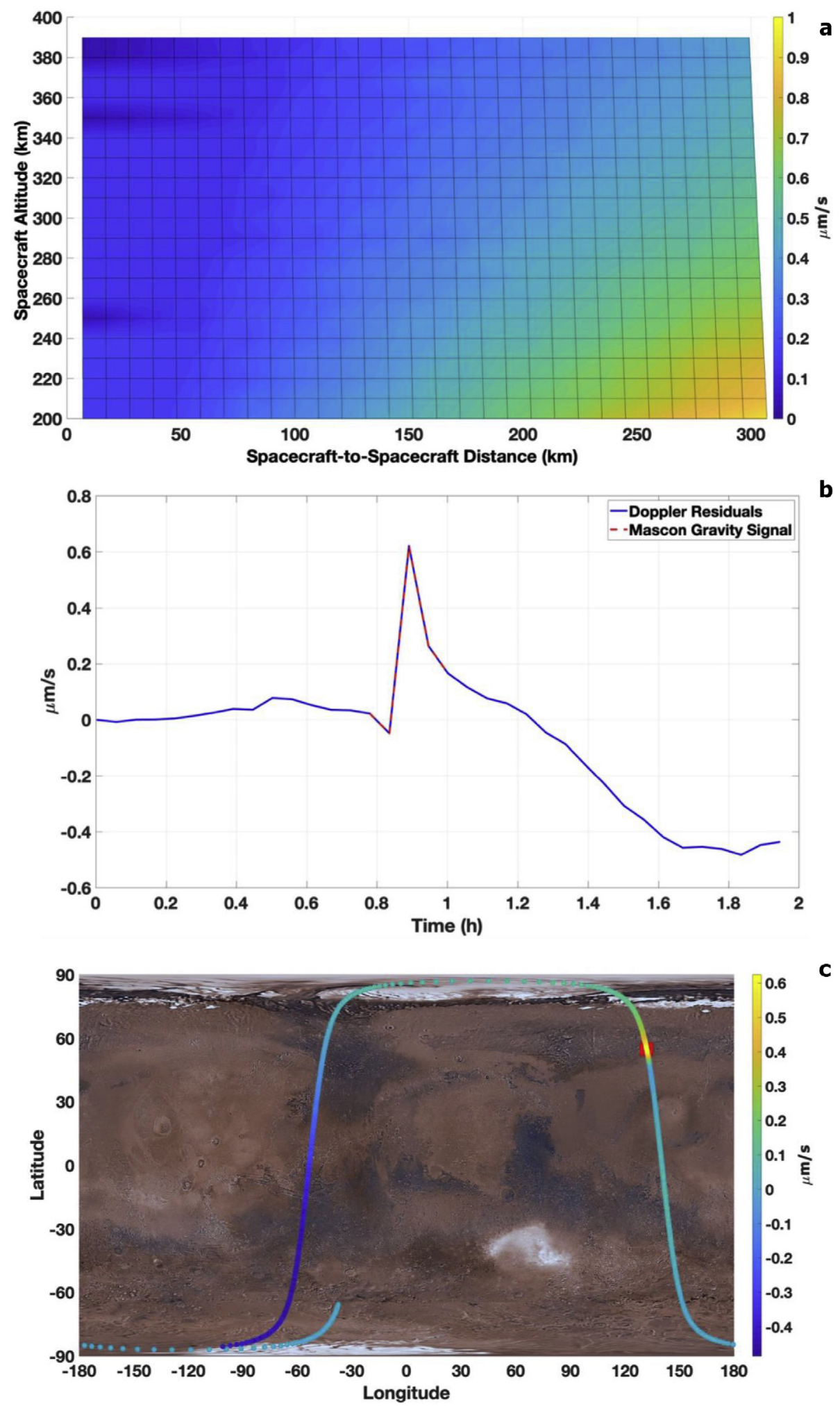

Fig. 2. Mascon gravity signal that is measured by simulated radio tracking data acquired at different altitudes and spacecraft-to-spacecraft distances (a). The mascon is modeled with a magnitude of $0.01 \mathrm{mGal}$, which is about the gravity signal associated with a $\mathrm{CO}_{2}$ ice slab of $\sim 30 \mathrm{~cm}$, and located at $55^{\circ} \mathrm{N}$ with a size of $360 \times 360$ $\mathrm{km}^{2}$. Radio tracking data residuals of the simulation at $\sim 285-\mathrm{km}$ altitude and $300-\mathrm{km}$ spacecraft-to-spacecraft distance are reported as a function of time (b) and in a latitude-longitude map (c). The time-series of the residuals shows the full period $(\sim 2$-h) and the time interval when the first spacecraft crosses the mascon area with blue and red curves, respectively. The peak in the red dashed curve represents the gravity signal that is measured with the tracking data. The map in the bottom panel shows the Martian surface, the Doppler residuals in colors, and the mascon as a red square. (For interpretation of the references to color in this figure legend, the reader is referred to the Web version of this article.) 
velocity between ORACLE-A and -B, if they act similarly on both spacecraft. The atmospheric drag represents the main perturbation and strongly depends on the properties of the two probes. Our spacecraft architetture accounts for similar ballistic coefficients $\frac{M_{1}}{C_{D 1} A_{1}} \cong \frac{M_{2}}{C_{D 2} A_{2}}$, where $C_{D i}, M_{i}$ and $A_{i}$ are the drag coefficient, mass and cross sectional area of each spacecraft $i$, respectively. This flight system configuration leads to small discrepancies in the spacecraft orbit evolutions due to the atmospheric drag. Similarly, the thermo-optical properties of the bus and the solar panels (e.g., reflectivities) are assumed to be identical between the two orbiters in order to mitigate the perturbations on the SST data induced by solar and albedo radiation pressures.

The spacecraft buses are based on a small satellite platform, whose mass is $\sim 40 \mathrm{~kg}$. The Electrical Power Subsystem (EPS) consists of 4- $m^{2}$-area solar panels, a Power Control and Distribution Unit (PCDU), and a $357 \mathrm{Whr}$ Li-Ion battery supply electrical power of $\sim 140 \mathrm{~W}$ to both spacecraft during eclipses. This power budget has been obtained by assuming the consumption of each spacecraft system and a $20 \%$ design margin for a conservative assessment. The thermal subsystem consists of radiators, blankets and heaters to have a passively controlled system. Errors induced by thermal changes may lead to unexpected variations in the location of the microwave horn phase center with respect to the spacecraft center-of-mass (antenna offset). The thermal stability of the spacecraft represents an important design constraint to enable a precise knowledge of the antenna offset over the course of the entire mission, which represents a key mission requirement. Mismodeling of the relative distance between microwave horn phase center and spacecraft center-of-mass causes undesired effects in the inter-satellite range-rate data.

Both spacecraft are three-axis stabilized with multi-head star tracker [39], two Sun sensors, three reaction wheels ( $4 \mathrm{~N} \mathrm{~m} \mathrm{~s}$ ), and an Inertial Mass Unit (IMU) that is also important for aerobraking operations [40]. The propulsion system assumed for both spacecraft includes two sets of four hydrazine thrusters $(4 \mathrm{~N})$ to control their trajectories through OCMs and to desaturate the reaction wheels. We considered an amount of propellant of $41.5 \mathrm{~kg}$ on each spacecraft that enables aerobraking control maneuvers, and orbit control and desaturation maneuvers for at least one entire Martian year. However, this mass does not account for propellant that would be necessary to place the spacecraft in a disposal orbit at the end of the mission. Because of the low-altitude orbit of the Mars ORACLE mission, spacecraft failure would lead to a premature impact before the long-term orbital lifetime ( $>50$ years) defined by the current planetary protection policy. The design of very clean spacecraft with a microbiological burden of $<50,000$ microbial spores, which requires manufacturing controls and cleaning, represents the plan of the Mars ORACLE mission to fulfill planetary protection requirements. This strategy that is in line with international space agencies regulations was adopted by the MRO mission [41].

The scientific instrumentation are allocated on ORACLE-A and -B taking into account the operational constraints and the need to have similar payload mass and power consumption on each spacecraft. Section 3.3 presents in detail the scientific payload that is assumed to have a mass $<15 \mathrm{~kg}$ and available power $<50 \mathrm{~W}$ for both probes.

\subsection{Scientific instrumentation}

The ORACLE mission concept includes three science payloads that are the RS system, TIR wide-angle camera, and laser altimeter (Table 3). The first two instruments are hosted by ORACLE-B, and the laser altimeter represents the only science instrumentation on ORACLEA since the TT\&C system is used for both SST and data relay function back to Earth. This dual-spacecraft configuration is well-suited to estimate accurately variations of intensity of Mars' gravity field by measuring the relative velocity between ORACLE-A and -B. The RS system architecture consists of TT\&C components that contribute to science (Integrated Deep Space Transponder, IDST [42]) and dedicated payload components (e.g, Ultra Stable Oscillator, USO, and transceiver). Fig. 3 shows the block diagram of the science payload and part of the TT\&C system units.

ORACLE-A enables data relay function to the DSN stations through a set of fixed front and back antennas (e.g., fan beam medium gain and phased array high-gain antennas [43]), and the IDST [42]. The deep space radio tracking data acquired $\sim 8 \mathrm{~h}$ per day by the DSN stations are in X-band only, leading to accuracies of $\sim 1 \mathrm{~m}$ for range and $\sim 0.1 \mathrm{~mm}$ $\mathrm{s}^{-1}$ for range-rate at $10^{-} \mathrm{s}$ integration time.

The IDST is also a key element for the SST in both X- and Ka-band. ORACLE-B transmit toward ORACLE-A a stable, Ka-band signal generated by a USO [44] and upconverted by a transceiver. An amplifier and a microwave horn complete the transmitting chain. ORACLE-A receives the signal and sends it back to ORACLE-B through the IDST that phases lock the retransmitted signal frequencies in X- and Ka-band for a simultaneous double-link. The transceiver receives the two-way signal and compares the outgoing and incoming frequencies to provide the SST range-rate observable. Due to the short round-trip light-time ( $\sim 2$ $\mathrm{ms})$ the USO instability, which has an Allan deviation $\left(\frac{\Delta f}{f}\right)$ of $10^{-12}$ at 1000 -s count interval, is largely suppressed. For 10-s integration time, the suppression factor is $\frac{10}{2 \times 10^{-3}}=5000$ leading to a resulting Allan deviation of $2 \times 10^{-15}$. The double-frequency measurements allow separating dispersive perturbations due to the ionosphere; this adds the capability to retrieve ionospheric density profiles.

This two-link frequency scheme enables SST range-rate data with a precision of $\sim 0.6 \mu \mathrm{m} \mathrm{s}^{-1}$ at $10^{-} \mathrm{s}$ integration time. These measurements are significantly different from the Ka-band range-rate (KBRR) data collected by the GRACE and GRAIL missions. The RS system of both NASA missions provided a dual-one-way ranging (DOWR) observation to precisely measure the relative motion between the two spacecraft. Ka-band ranging (KBR) measurements were generated through sophisticated instruments including an USO, a microwave assembly, and a processor unit on each spacecraft. The time synchronization between spacecraft clocks and USOs represented the main issue for DOWR configuration. The GRACE spacecraft hosted Global Positioning System (GPS) receivers for precise time-tagging of the KBR measurements [45]. The GRAIL spacecraft, on the other hand, used a time transfer unit that enabled an additional inter-satellite link in S-band to synchronize and measure clocks offsets [46]. The Mars ORACLE RS system is not affected by the synchronization of the spacecraft clocks since it takes advantage of the IDST to generate directly two-way range-rate measurements. This scheme allows to significantly reduce the number of the onboard RS elements leading to measurements that are only a factor of $\sim 3$ less accurate compared to GRACE and GRAIL data.

The radio system instrumentation relies on solid technology heritages of NASA missions Cassini [47] and Juno [48], and ESA missions BepiColombo [49] and JUpiter ICy moons Explorer (JUICE) [50]. The IDST, whose Engineering Model (EM) has being developed under the General Support Technology Programme (GSTP), is configured to operate at the X- and Ka-band frequencies used for deep space communications. On 13 September 2017, ESA Consultative Committee for Space Data System (CCSDS) provided frequency assignment guidelines for communications in the Mars region through the Recommendation of the Space Frequency Coordination Group (SFCG) [51]. The recommended inter-satellite Ka-band frequencies of Mars' orbit-to-orbit scenario are $22.55-23.55 \mathrm{GHz}$ and $25.5-27 \mathrm{GHz}$ that differ from the KaBand frequencies used for deep space $(\sim 32 \mathrm{GHz})$. The fulfilment of this recommendation would require, therefore, a requalification of the IDST at those frequency bands leading to a lower Technology Readiness Level (TRL) compared to the developed EM.

ORACLE-B also hosts a second science instrument that is a TIR wideangle camera to track the extension of the caps edges by creating image mosaics of the polar regions. One of the main advantages of IR wavelengths is a better contrast between $\mathrm{CO}_{2}$ ice from a retreating annulus of $\mathrm{H}_{2} \mathrm{O}$ ice. Piqueux et al. [22] showed an accurate mapping of the polar 
Table 3

Scientific Payload and TT\&C instruments for ORACLE-A and -B.

\begin{tabular}{|c|c|c|c|c|c|c|}
\hline & Science Investigation & Instrument* & Mass (kg) & Power $(W)$ & TRL & Heritage \\
\hline$\ll$ & \multirow{3}{*}{ Radio Science } & $\mathrm{X} / \mathrm{Ka}-\mathrm{B}$ and Amplifier & 1 & 1 & $6-7$ & BepiColombo-JUICE \\
\hline \multirow{3}{*}{ 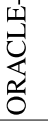 } & & Microwave Horn & 3 & - & $7-8$ & GRAIL \\
\hline & & Integrated DST & 3 & 20 & $5-6$ & BepiColombo-JUICE \\
\hline & Altimetry & Laser Altimeter & 8 & 25 & $6-7$ & MLA, LOLA, BELA \\
\hline \multirow{5}{*}{ 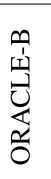 } & \multirow{4}{*}{ Radio Science } & Transceiver & 3 & 20 & $6-7$ & BepiColombo-JUICE \\
\hline & & USO & 2.5 & 2.2 & 9 & GRAIL-JUICE \\
\hline & & Ka Amplifier & 1 & 10 & $6-7$ & BepiColombo-JUICE \\
\hline & & Microwave Horn & 3 & - & $7-8$ & GRAIL \\
\hline & Imaging & TIR Camera & 3.5 & 15 & $6-7$ & Hayabusa2 \\
\hline
\end{tabular}

Instruments in black are elements exclusively dedicated to science investigations. TRL are based on the solid heritage of existing NASA and ESA instruments.

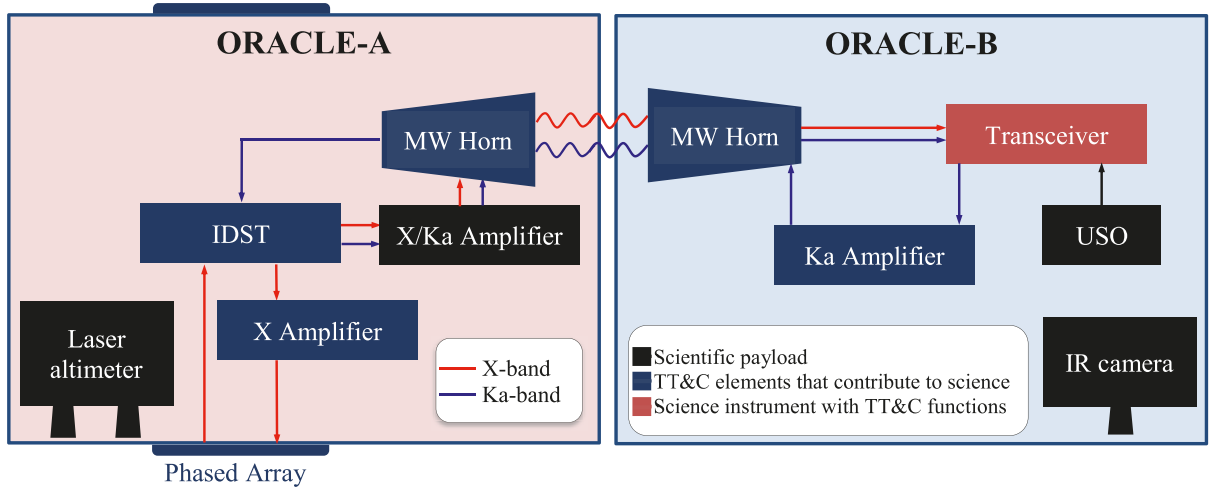

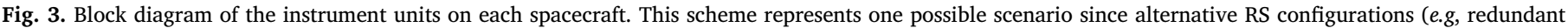
transponder) are still consistent with medium-class mission boundary constraints.

Table 4

Instruments performances and measurements requirements of the Mars ORACLE mission that account for the objectives of the scientific investigations.

\begin{tabular}{|c|c|c|c|}
\hline Scientific Payload & Instrument Performances & Measurement Requirements & Mission Objectives \\
\hline RS system & $\begin{array}{l}1 \mathrm{~m} \text { range }-0.1 \mathrm{~mm} \mathrm{~s}^{-1} \text { range-rate } @ 10^{-} \mathrm{s} \\
(\text { Deep space tracking) } \\
0.6 \mu \mathrm{m} \mathrm{s}^{-1} \text { range-rate } @ 10^{-} \mathrm{s}(S S T)\end{array}$ & $\begin{array}{l}3 \mathrm{~m} \text { range }-0.3 \mathrm{~mm} \mathrm{~s}^{-1} \text { range-rate } @ 10^{-} \mathrm{s} \\
\text { (Deep space tracking) } \\
1 \mu \mathrm{m} \mathrm{s}^{-1} \text { range-rate } @ 10^{-} \mathrm{s}(S S T)\end{array}$ & $\begin{array}{l}\text { Spacecraft position accuracies of }<1 \mathrm{~m} \text { radially, and } 10-50 \\
\mathrm{~m} \text { along- and cross-track } \\
\text { Gravity anomaly accuracies of } 0.015 \mathrm{mGal} \text { on } 360 \times 360 \mathrm{~km}^{2} \\
\text { local regions over an entire Martian season }\end{array}$ \\
\hline Laser Altimeter & $30 \mathrm{~cm}$ radially & $50 \mathrm{~cm}$ radially & $\mathrm{CO}_{2}$ ice slab thickness accuracies of $50 \mathrm{~cm}$ \\
\hline TIR camera & $\begin{array}{l}200-300 \mathrm{~m} \text { spatial resolutions (IFOV of } \\
1 \mathrm{mrad} \text { ) with a FOV of } 65^{-} 85 \mathrm{~km}\end{array}$ & $\begin{array}{l}1-2 \mathrm{~km} \text { spatial resolutions with a FOV of } \\
100^{-} 150 \mathrm{~km}\end{array}$ & $\begin{array}{l}\text { Mapping the seasonal polar cap edges with an accuracy of } \\
\text { few kilometers }\end{array}$ \\
\hline
\end{tabular}

caps edges with MRO Mars Climate Sounder (MCS) thermal infrared data. This instrument is more complex with one channel in the visible and near infrared range $(0.3-3.0 \mu \mathrm{m})$ to investigate the effects of the solar energy on the atmosphere and the surface, and eight channels in the TIR range $(12-50 \mu \mathrm{m})$ to measure temperature, pressure, water vapor, and dust. MCS measurements at $\sim 32 \mu \mathrm{m}$ wavelengths have been used to define accurately the edges of the polar caps. This spectral range has the advantage over visible/near infrared data to be independent from illumination conditions. The camera of the Mars ORACLE mission concept enables images of thermal infrared emission between 5 and 15 $\mu m$ wavelengths with a field of view (FOV) of $\sim 15^{\circ} \times 15^{\circ}$ and an instantaneous field of view (IFOV) of $0.05^{\circ}$ that guarantees spatial resolutions of $\sim 200-300 \mathrm{~m}$. The TIR camera always points nadir to provide a detailed mosaic of images in both polar caps. The performances of this instrument allow to cover a temperature range from 150 to $350 \mathrm{~K} \mathrm{[52],} \mathrm{which} \mathrm{is} \mathrm{adequate} \mathrm{to} \mathrm{monitor} \mathrm{the} \mathrm{seasonal} \mathrm{extension} \mathrm{of}$ the polar caps.

To measure the total volume of the polar caps, the thickness of the seasonal deposits is determined by using a laser altimeter onboard ORACLE-A. This instrument inherits from the successful laser altimeters (e.g., MOLA [23], Lunar Orbiter Laser Altimeter, LOLA [53], BepiColombo Laser Altimeter, BELA [54]) developed for ESA and NASA missions to terrestrial planets. Its main components are a laser transmitter, receiver telescope, aft optics, detector, electronics, and microprocessor for instrument control and data processing [55]. The ORACLE laser altimeter operates at $\sim 1064 \mathrm{~nm}$ wavelength, and it is based on Mercury Laser Altimeter (MLA) of the MErcury Surface, Space ENvironment, GEochemistry, and Ranging (MESSENGER) mission that had a size of $30 \times 30 \times 30 \mathrm{~cm}^{3}$, a mass of $\sim 7.4 \mathrm{~kg}$, and the electrical power consumption while in science measurement of $\sim 23 \mathrm{~W}$ [56]. This instrument also guarantees high performance with laser pulse energy of $\sim 20 \mathrm{~mJ}$, pulse repetition rate of $9 \mathrm{~Hz}$ enabling highly accurate range to Mars surface with a vertical precision of $\sim 30 \mathrm{~cm}$.

Table 4 presents a comparison between the performances of the instruments onboard ORACLE-A and -B and the measurement requirements that enable the fulfillment of the mission objectives. The deep space tracking data provide an accurate estimation of the spacecraft position in the Martian reference frame. The quality of the orbital reconstruction significantly affects the operations of both laser altimeter and camera since mismodeling of the spacecraft position would lead to 


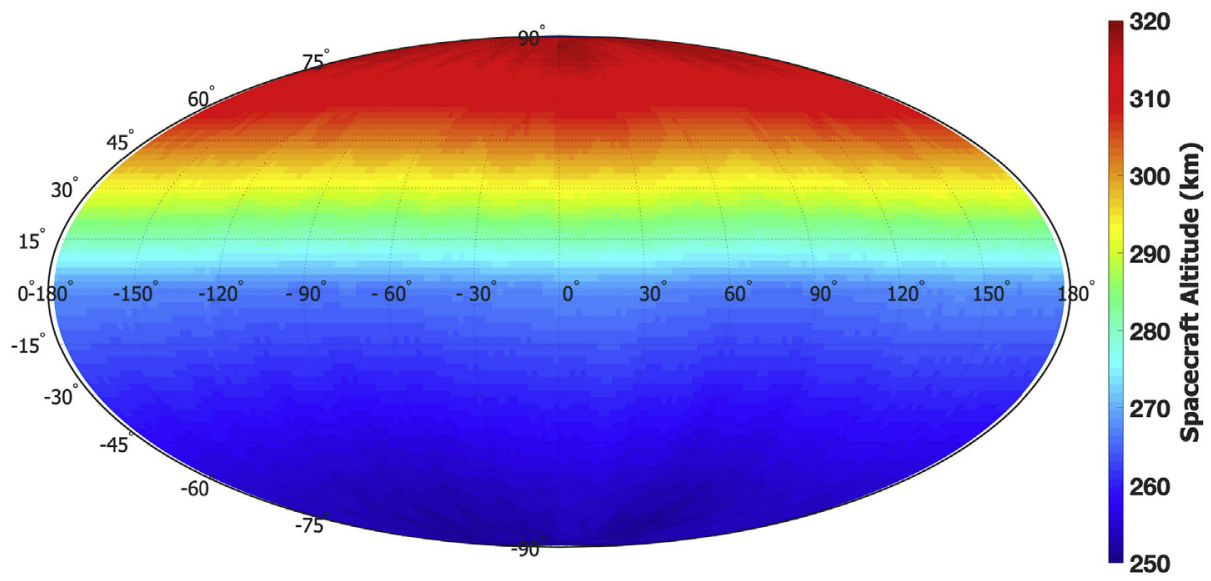

Fig. 4. ORACLE spacecraft altitudes with respect to the Martian ellipsoid with $3376 \mathrm{~km}$ and $3396 \mathrm{~km}$ radii at the poles and equator, respectively.

errors in altimetric and imaging data georeferencing. Accuracies of $3 \mathrm{~m}$ and $0.3 \mathrm{~mm} \mathrm{~s}^{-1} @ 10 \mathrm{~s}$ for range and range-rate data, respectively, are fully adequate to yield spacecraft positions with uncertainties of $<1 \mathrm{~m}$ radially, and 10-50 $\mathrm{m}$ along- and cross-track. The SST range-rate measurement requirement is $1 \mu \mathrm{m} \mathrm{s}^{-1}$ that allows to detect $\sim 0.015$ mGal gravity anomalies variations on $360 \times 360^{-} \mathrm{km}^{2}$ local polar regions during an entire Martian season. This gravity uncertainty corresponds to an accumulation of $\mathrm{CO}_{2}$ ice of $\sim 50 \mathrm{~cm}$, which represents the measurement specification for the laser altimeter. Typical radial precisions of single-beam laser altimeters are of the level of $30 \mathrm{~cm}$. The determination of the volume of the polar deposits also relies on the TIR camera spatial resolution, which has to be $<1-2 \mathrm{~km}$. This level of precision is suitable for accurately mapping of the seasonal polar cap edges.

\section{Geophysical and geodetic model estimation}

To define the requirements of the Mars ORACLE mission, a set of numerical simulations of the gravity and altimetry experiments were carried out accounting for constraints related to the orbital configuration. The determination of the geophysical and geodetic quantities relies on the data quality (Section 3.3) and coverage (Section 4.1), and on errors due to mismodeling of the orbital dynamics (Section 4.2). The impact of these key factors on the mission science objectives is investigated through numerical simulations based on an accurate modeling of the gravitational and topographic fields, and the perturbative forces including solar, albedo, and infrared radiation pressure and atmospheric drag. The gravitational potential $(U)$ and the topography $(T)$ of Mars were modeled as spherical harmonic expansions [57], as follows:

$$
\begin{gathered}
U=\frac{G M}{r}\left\{1+\sum_{l=2}^{l_{\max }}\left(\frac{a_{e}}{r}\right)^{l} \sum_{m=0}^{l}\left[\bar{C}_{l m}^{g} \cos m \lambda+\bar{S}_{l m}^{g} \sin m \lambda\right] \bar{P}_{l m}(\sin \varphi)\right\} \\
T=\sum_{l=0}^{l_{\max }^{t}} \sum_{m=0}^{l}\left[\bar{C}_{l m}^{t} \cos m \lambda+\bar{S}_{l m}^{t} \sin m \lambda\right] \bar{P}_{l m}(\sin \varphi)
\end{gathered}
$$

where $G M=42828.37 \mathrm{~km}^{3} \mathrm{~s}^{-2}$ is the gravitational parameter of Mars, $a_{e}=3396 \mathrm{~km}$ is the reference equatorial radius, $\bar{P}_{l m}$ are the normalized associated Legendre functions of degree $l$ and order $m$, and $r, \lambda$, and $\varphi$ are the body-fixed spherical coordinates of radial distance, longitude, and latitude, respectively. The normalized spherical harmonic coefficients for the gravity field and topography are $\bar{C}_{l m}^{g}$ and $\bar{S}_{l m}^{g}$, and $\bar{C}_{l m}^{t}$ and $\bar{S}_{l m}^{t}$, respectively. The relationship between normalized and unnormalized coefficients is:

$\left(\begin{array}{l}C_{l m} \\ S_{l m}\end{array}\right)=\left[\frac{(2 l+1)\left(2-\delta_{0 m}\right)(l-m) !}{(l+m) !}\right]^{\frac{1}{2}}\left\{\begin{array}{l}\bar{C}_{l m} \\ \bar{S}_{l m}\end{array}\right\}$ where $C_{l m}$ and $S_{l m}$ are the unnormalized coefficients and $\delta_{0 m}$ is 1 if $m=0$, and is zero otherwise [57].

The latest solution of the Martian global topography based on the MOLA measurements has a spatial resolution of $\sim 9 \mathrm{~km}$ that corresponds to degree and order 1152 in spherical harmonics [23,58]. The laser altimeter onboard the Mars ORACLE mission is designed to enhance our knowledge of the Martian topographic relief yielding an accurate estimation of spherical harmonic coefficients to degree and order 1500. The numerical simulations of the altimetry investigation are conducted by assuming the Moon's topography archived at http://pds-geosciences. wustl.edu/lro/lro-l-lola-3-rdr-v1/lrolol_1xxx/data/lola_shadr/, [59] as a priori field that has a spatial resolution on Mars of $\sim 5 \mathrm{~km}(l=2050)$.

The Goddard Gravity Model (GMM) - 3 is the latest gravity solution that was retrieved by processing the full MGS dataset, Mars Odyssey data from 2002 to 2015, and MRO data from 2006 to 2015 [20,26]. The numerical simulations of the ORACLE mission were based on the assumption that the a priori gravity field, which was adopted to generate the simulated data, was GMM-3 up to degree $l=120$ and MOLA topography-induced gravity for higher degrees $(l=120-150)$.

\subsection{Data coverage}

The spacecraft orbit configuration strongly affects the outcome of both gravity and altimetry experiments. The spacecraft altitude, in particular, represents a key element for the gravity measurements. The radio signal associated to gravity anomalies is larger at lower altitudes leading to higher signal-to-noise ratios. Fig. 4 shows ORACLE-A altitudes on Mars' surface during SST data acquisition. Because of the orbit eccentricity and the periapsis located in the southern hemisphere $\left(\omega_{p}\right.$ $=270^{\circ}$ ), the spacecraft altitude is $\sim 250 \mathrm{~km}$ and $\sim 320 \mathrm{~km}$ over the south and north polar regions, respectively. Therefore, the achievable gravity accuracies are significantly enhanced in the southern hemisphere.

The orbit configuration also affects the surface coverage of the laser altimeter data. Fig. 5 shows the number of laser altimeter observables on the Martian surface, which is discretized with a pixel size of $0.12^{\circ}$ ( $\sim 7 \mathrm{~km}$ at the equator). The poles are uniformly covered with a number of observables between 80 and 100 up to latitudes $87^{\circ}$ in both hemispheres. The equatorial region also shows a fair number of laser measurements between 50 and 60 with few gaps that have 10-20 data points only. To simulate this data coverage, we assumed that the laser altimeter operates $\sim 16 \mathrm{~h}$ per day continuously with a pulse repetition rate of $9 \mathrm{~Hz}$ with a random schedule that varies day by day. The simulated data also account for false detection rate of $1 \%$.

Both altimetry and time-variable gravity measurements depend on the ground track patterns of the Mars ORACLE mission. In this study, we considered the MRO orbit that is near-polar, near-circular, and midafternoon (3 p.m.) sun-synchronous. This orbital configuration was 


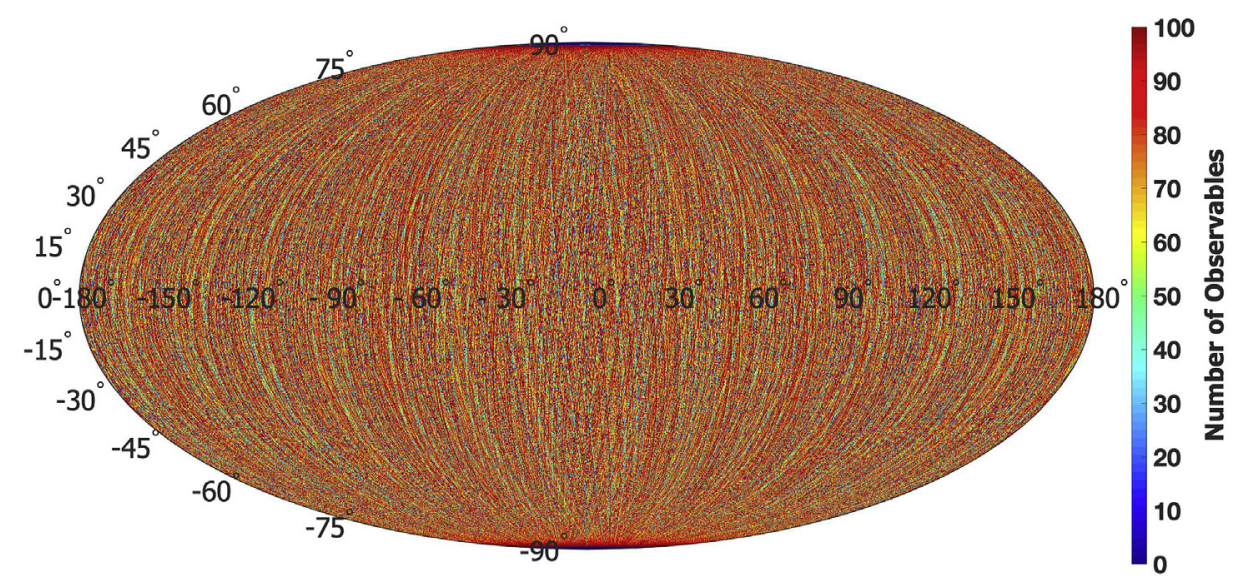

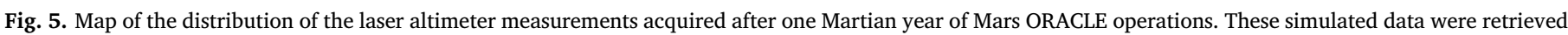
by assuming $16 \mathrm{~h}$ per day operations, a pulse repetition rate of $9 \mathrm{~Hz}$, and a false detection rate of $1 \%$.

designed to enable global mapping, regional survey, and target observations with the MRO science payload [37] including the gravity investigation [16] and the shallow radar (SHARAD) [60]. The assumption that the Mars ORACLE spacecraft follow the MRO orbit represents a suitable initial reference. However, ORACLE-A and -B orbit configuration may be opportunely adjusted to significantly improve their ground track distribution. Enhancements of the Martian surface coverage allow, for example, to avoid undesired evolution of the timevariable gravity accuracy that was seen by the GRACE investigation [61].

\subsection{Orbital dynamics errors}

A precise reconstruction of the spacecraft trajectories represents a stringent requirement for the scientific investigations of the Mars ORACLE missions. Mismodeling of non-conservative forces may cause accelerations that severely affect the orbital dynamics. The accelerations that mainly perturb the spacecraft orbit are the atmospheric drag, solar radiation pressure, Mars' albedo and thermal emission, and spacecraft thermal radiation. These perturbations were included in the numerical simulations of the Mars ORACLE mission, and both spacecraft were modeled with seven single-side flat plates by assuming six panels for the bus and one $4-\mathrm{m}^{2}$ solar array. Thermo-optical properties and drag coefficient $\left(C_{D}\right)$ of the spacecraft panels were based on MRO values. The effects of those undesired accelerations are mitigated by considering similar ballistic coefficients of the two probes (Section 3.2).

The main perturbation acting on both spacecraft is the atmospheric drag that varies accordingly with Mars' local solar time (LST) and season. The drag acceleration is computed as follows:

$\boldsymbol{a}_{\boldsymbol{D}}=\frac{1}{2} \frac{A C_{D}}{m} \rho V \boldsymbol{V}$

where $\rho$ is the Martian atmospheric density $\left(1 \cdot 10^{-13}-2 \cdot 10^{-12} \mathrm{~kg} \mathrm{~m}^{-3}\right)$, $A, C_{D}, m$, and $\boldsymbol{V}$ are the cross-sectional area $\left(\sim 5 \mathrm{~m}^{2}\right)$, drag coefficient $(\sim 2.2)$, mass $(\sim 215 \mathrm{~kg})$, and velocity vector of the spacecraft $\left(\sim 3.5 \mathrm{~km} \mathrm{~s}^{-2}\right)$, respectively. Large values of the atmospheric densities lead to accelerations of $\sim 600 \mathrm{~nm} \mathrm{~s}^{-2}$. An accurate modeling of the Martian atmosphere in the orbit determination software is then needed to compute the predicted values of the atmospheric densities. Our numerical simulations were carried out by assuming the Drag Temperature Model $^{-}$Mars (DTM-Mars) [62] as the reference atmospheric model in our orbit determination software.

We investigated the effect on the radio tracking data induced by possible errors in the predicted atmospheric densities. The inter-satellite range-rate measurements are particularly sensitive to the differential contribution of the non-gravitational forces on the two spacecraft. Fig. 6-a shows the discrepancies in the atmospheric drag accelerations between ORACLE-A and -B during SST data acquisition over an entire Martian year. Since the two spacecraft are $300 \mathrm{~km}$ apart, the distinct atmospheric densities at ORACLE-A and -B locations lead to different atmospheric drag forces. We considered $50 \%$ errors in the Martian atmospheric model predictions to determine these effects in the SST range-rate data. Fig. 6-b shows that the assumed atmospheric mismodeling generate SST signals that are always lower than the instrument precision $\left(0.6 \mu \mathrm{m} \mathrm{s}^{-1} @ 10 \mathrm{~s}\right)$. During summer in the southern hemisphere, there is a clear peak due to the higher atmospheric densities compared to other seasons. The signal is $<0.1 \mu \mathrm{m} \mathrm{s}^{-1}$ for $75 \%$ of the time reaching values close to $0.4 \mu \mathrm{m} \mathrm{s}^{-1}$ for $L_{s}$ close to $270^{\circ}$ (summer solstice in the southern hemisphere). Our results are conservative since we accounted for a powerful dust storm season and a constant intense solar activity (solar flux at $10.7 \mathrm{~cm}, \mathrm{~F} 10.7$, and 1 Astronomical Unit, AU, of 175 solar flux unit, sfu) in our atmospheric model. Nevertheless, the atmospheric perturbative signal is still within the instrument noise and the presented radio science instrumentation is well-suited to address the scientific goals regarding the time-variable gravity.

Drag effects, however, provide errors that accumulate over time leading to discrepancies in the spacecraft orbit reconstruction. The laser altimeter onboard ORACLE-A requires accuracies in the along- and cross-track directions of few tens of meters. Dedicated deep space radio tracking passages (8-h per day) with ORACLE-A enable highly accurate orbit determination solutions that are retrieved by adjusting one atmospheric drag coefficient for each spacecraft orbit [20]. The estimation of these coefficients, which are used as scale factors of the atmospheric drag forces, helps to mitigate spacecraft orbital errors leading to the required accuracies.

The other main perturbations acting on the spacecraft are associated with the radiation pressure due to the Sun and Mars, and the thermal re-radiation of the spacecraft. The acceleration due to the solar radiation pressure has a maximum value on the order of $100 \mathrm{~nm} \mathrm{~s}^{-2}$ that is separable from the gravitational effects as demonstrated by the GRAIL mission [63]. The accelerations induced by spacecraft thermal radiation and Mars radiation pressure are between one and two orders of magnitudes lower compared to the solar radiation pressure. However, differences between ORACLE-A and -B temperatures and locations provide differential accelerations that affect the orbit propagation of the spacecraft orbits. Once-per-revolution periodic accelerations are then adjusted to absorb errors in the radiation pressure models including dust storm events that affect the modeling of Mars' umbra and penumbra [20].

The inclusion of an accelerometer onboard each spacecraft still represents a concrete opportunity. The solid technology heritage of this instrument, which is dedicated to the measurement of non-gravitational 

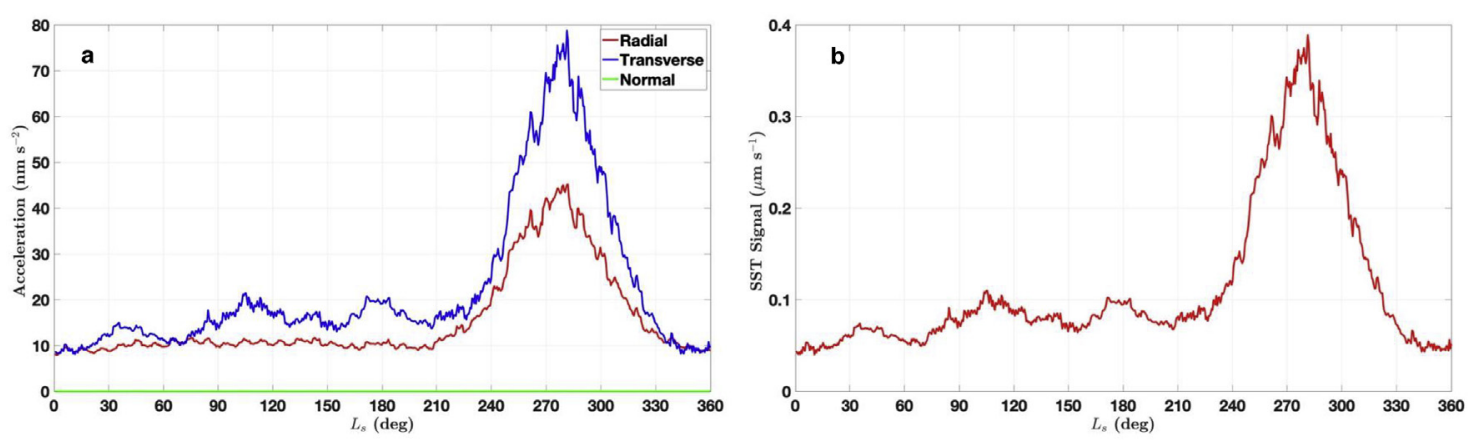

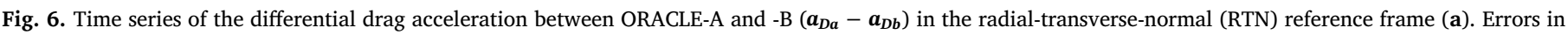

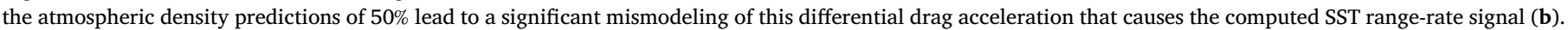

forces, is based on the three-axis electrostatic accelerometers onboard GRACE (SuperSTAR, [64]) and the three-axis torsional oscillator accelerometer onboard BepiColombo (Italian Spring Accelerometer, [65]). This additional payload, however, would complicate the proposed mission architecture leading to higher total cost, mass, and power demand.

\subsection{Mass and volume of the seasonal polar caps}

The numerical simulations of the Mars ORACLE mission were mainly focused on the determination of the mass and thickness of the polar deposits with gravity and altimetry data, respectively. We simulated the inter-satellite tracking data collected during the first 180 days (an entire Martian season) of the Mars ORACLE mission. The inter-satellite range-rate data were assumed to be acquired continuously with a conservative level of noise of $1 \mu \mathrm{m} \mathrm{s}^{-1} @ 10 \mathrm{~s}$ (Section 3.3). The gravity signal associated with the variations of $\mathrm{CO}_{2}$ seasonal polar deposits was modeled with mass concentrations (mascons). This approach was used for GRACE data analysis to observe Greenland ice mass loss [66]. The polar caps are then uniformly covered by equal-area mascons $\left(180 \times 180 \mathrm{~km}^{2}\right)$ between $55^{\circ}$ and $90^{\circ}$ latitudes in both hemispheres. The goal of our gravity simulations was the estimation of the mascons, which are adjusted with other parameters (e.g., spherical harmonic coefficients of the global gravity field) in a least-squares solution, to determine the achievable accuracies of the mass anomalies due to the seasonal polar deposits. Fig. 7a-b shows the resulting mascon uncertainties $\left(\sigma_{M}\right)$ for latitudes between $65^{\circ}$ and $90^{\circ}$ in both hemispheres by computing $\sigma_{M}=\frac{\sigma_{g} A_{M}}{2 \pi G}$, where $\sigma_{g}$ is the retrieved gravity uncertainty and $A_{M}$ is the area of the mascon $\left(\sim 180 \times 180 \mathrm{~km}^{2}\right)$. Our gravity analysis shows accuracies of $<1.2 \times 10^{13} \mathrm{~kg}$ that correspond to $\sim 0.015 \mathrm{mGal}$ in gravity anomalies and $\sim 40 \mathrm{~cm}$ accuracies for $\mathrm{CO}_{2}$ thickness variations.

Numerical simulations of the altimetry experiment were also carried out to determine the achievable accuracy of the $\mathrm{CO}_{2}$ ice slab thickness. During each season, the laser altimeter provides multiple laser shots over the same location at different Martian days. These data measure the radius differences between ground track pairs at their common intersection (altimetric crossovers) [67]. The vertical discrepancy of each crossover is a direct measurement of the temporal changes in the elevation of the Martian surface associated with seasonal deposits of the polar caps [5]. We simulated the laser altimeter crossovers data to determine the resulting thickness uncertainties of the accumulated $\mathrm{CO}_{2}$ ice $\left(\sigma_{h}\right)$. Fig. $7 \mathrm{c}-\mathrm{d}$ shows the solution of the crossover analysis by computing $\sigma_{h}=\frac{\sigma_{x}}{\sqrt{N_{x}}}$ where $\sigma_{x}$ and $N_{x}$ are the measurement precision and the number of crossovers, respectively. A conservative measurement precision of $1 \mathrm{~m}$ was assumed to determine $\sigma_{h}$. The number of crossovers was obtained over the surface regions defined by each gravity mascon.

The results of our gravity and crossover analysis suggest that the proposed radio science and laser altimeter instrumentation provide accurate observations of $\mathrm{CO}_{2}$ ice mass anomalies and thickness, respectively. Because of the orbit configuration, the gravity measurements are much more accurate in the southern hemisphere. Fig. 7a-b also show a longitudinal pattern that is due to the modeling of the equiarea mascons. To reduce the larger uncertainties at lower latitudes, different modeling of the mascons can be adopted as, for example, equiangular mascons [68]. However, the results of the gravity simulations demonstrate that a finer sampling of the mascon grid is still adequate to measure gravity anomalies of $\sim 0.015 \mathrm{mGal}$. The laser altimeter crossovers also yield enhanced measurements of the thickness of the seasonal deposits close to the poles. Few areas at latitudes lower than $75^{\circ}$ show $\sigma_{h}$ of $\sim 30 \mathrm{~cm}$ that is still in line with the uncertainties achievable with the measured mass anomalies from gravity. The Mars ORACLE PSP orbit, however, can be improved to provide a more uniform longitudinal coverage of the laser altimeter shots.

\subsection{Gravity and topographic maps}

High-resolution maps of the static gravity field and topography of Mars represent important objectives of the Mars ORACLE mission. The numerical simulations of the gravity and altimetry investigations were extended for an entire Martian year to assess the achievable accuracies with the orbital and instrument configurations presented in Section 3. The radio tracking data of the Mars ORACLE mission enable highly accurate estimates of the Martian gravity field coefficients beyond degree and order 150, which corresponds to a spatial resolution of $\sim 70$ $\mathrm{km}$ that represent a significant improvement compared to our current knowledge [20,26]. Fig. 8 shows the power spectrum of the gravity solutions that were obtained after one Martian season and year. The deep space radio tracking data collected 8-h per day enable an accurate measurement of the low-degrees, and the SST data provide substantial constraints on the higher degrees that define the short-wavelengths of the gravity anomalies.

The global topography of Mars measured by MOLA is defined in spherical harmonics to degree and order 1152 [23]. The laser altimeter onboard the Mars ORACLE mission can improve the spatial resolution of this global map. Therefore, the simulations of the altimetric dataset were conducted by assuming the Moon's topography [59], which is expanded in spherical harmonics to degree and order 2050, as a priori topography of Mars. The laser altimeter data were simulated by assuming a random daily operation schedule of $16 \mathrm{~h}$, a pulse repetition rate of $9 \mathrm{~Hz}$, and a false detection rate of $1 \%$. The total number of laser altimeter shots collected after one Martian year is $\sim 350$ M. Fig. 9 shows the topographic relief obtained through the simulations of the ORACLE laser altimeter measurements. The three local regions in the figure display the lunar landing sites of the Apollo 11, 12, 14 and 17 missions. The resolution of the resulting topographic map is significantly improved compared to the MOLA global map, which is represented in Fig. 9 by expanding the Moon's topography to degree and order 1152 . The Mars ORACLE mission enables the retrieval of the Martian global topography with a spatial resolution of $\sim 7 \mathrm{~km}(l=1500)$. The 

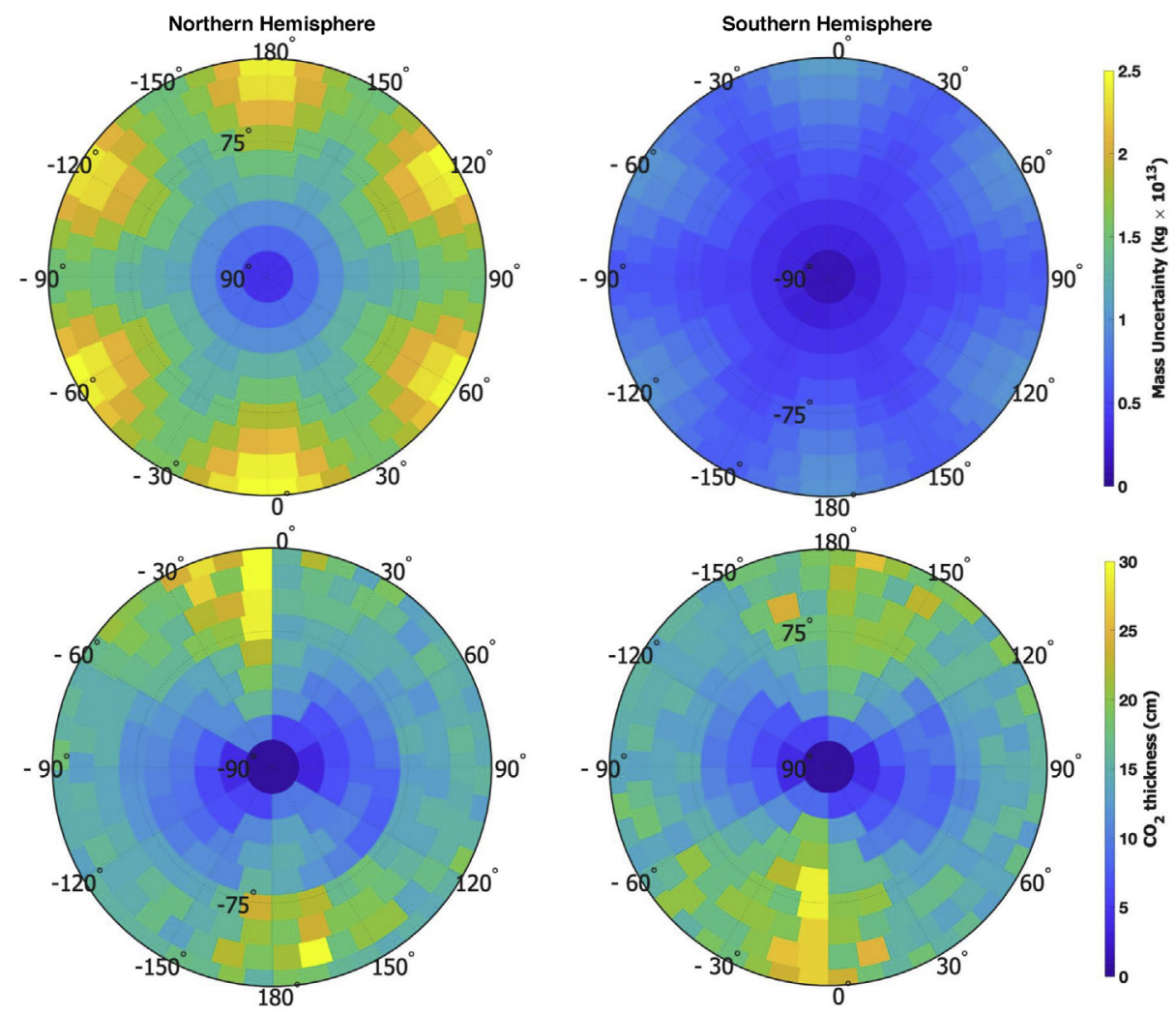

Fig. 7. Mass (a-b) and $\mathrm{CO}_{2}$ thickness (c-d) uncertainty of the north and south seasonal polar deposits achievable every Martian season ( 180 days) with ORACLE gravity and altimetry investigations, respectively. Each pixel represents a modeled mascon with an area of $180 \times 180 \mathrm{~km}^{2}$. A mass uncertainty of $0.9 \times 10^{13} \mathrm{~kg}$ corresponds to $\sim 0.01 \mathrm{mGal}$ of gravity anomaly (or to $\sim 30 \mathrm{~cm}$ of $\mathrm{CO}_{2}$ thickness by assuming $\rho \sim 900 \mathrm{~kg} \mathrm{~m}^{-3}$ ). The number of crossovers are computed on each mascon area, and the $\mathrm{CO}_{2}$ thickness uncertainties are $\sigma_{h}=\frac{\sigma_{x}}{\sqrt{N_{x}}}$ with $\sigma_{x}$ and $N_{x}$ are the measurement precision and the number of crossovers, respectively.

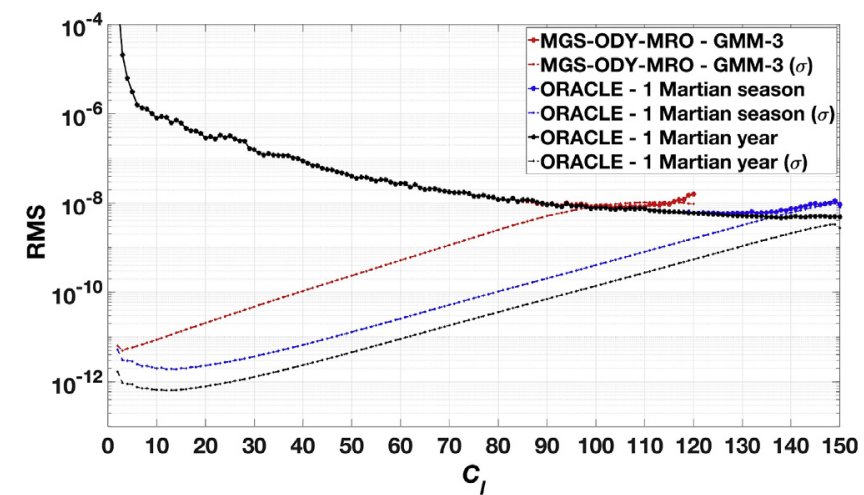

Fig. 8. Power spectra of GMM-3 solution (red), and the gravity solutions from two numerical simulations of the ORACLE mission that account for 1 Martian season (blue) and 1 Martian year (black) science operations. (For interpretation of the references to color in this figure legend, the reader is referred to the Web version of this article.)

combination of this enhanced global map with existing and future highresolution images will be pivotal to determine safe landing sites for future robotic and human missions.

\section{Conclusions}

Mars ORACLE is a mission concept dedicated to address fundamental open questions regarding the Martian climate and interior structure. We presented here a preliminary mission design that accounts for the boundary conditions of a medium-class mission of international space agencies. The mission configuration consists of three scientific instruments (i.e., RS system, laser altimeter and IR camera) that are based on solid technology heritages (TRL $>5-6$ ) leading to shortterm development plans ( $\sim 4-5$ years).

Other instrument configurations may be considered, for example, to determine the volume of the seasonal polar deposits. A TIR spectrometer could be well-suited to map the extension of the polar caps and characterize the properties of the polar deposits with enhanced accuracies, but this instrument would complicate significantly the mission profile because of its mass and power demand. A radar sounder could be a valuable alternative of the laser altimeter to measure seasonal variations of $\mathrm{CO}_{2}$ thickness. Its capability of radar echo sounding could also provide information on the presence of liquid water underneath the thick polar layered deposits [69]. However, the power demand and mass of a radar sounder significantly exceed the budgetary constraints of a medium-class mission. A mission configuration of Mars ORACLE with a scientific payload that consists of a RS system, a TIR spectrometer, and a radar sounder may be considered for ESA and NASA largeclass mission calls.

The baseline of the Mars ORACLE mission concept relies on the RS instrumentation, laser altimeter and TIR camera presented in Section 3.3. This scientific payload is fully adequate to accomplish the scientific goals presented in this study including the monitoring of the seasonal variations of Mars' polar caps. The total mass and power budgets of the mission shown in Table 2 are consistent with medium-class mission guidelines. However, the Cost at Completion $(\mathrm{CaC})$ also represents one of the main constraints of this mission configuration and profile. The flight system and mission operations are compliant with the cost cap of a medium-class mission. External contributions may be necessary to fully support the Mars ORACLE mission, especially, to design the scientific payload. 

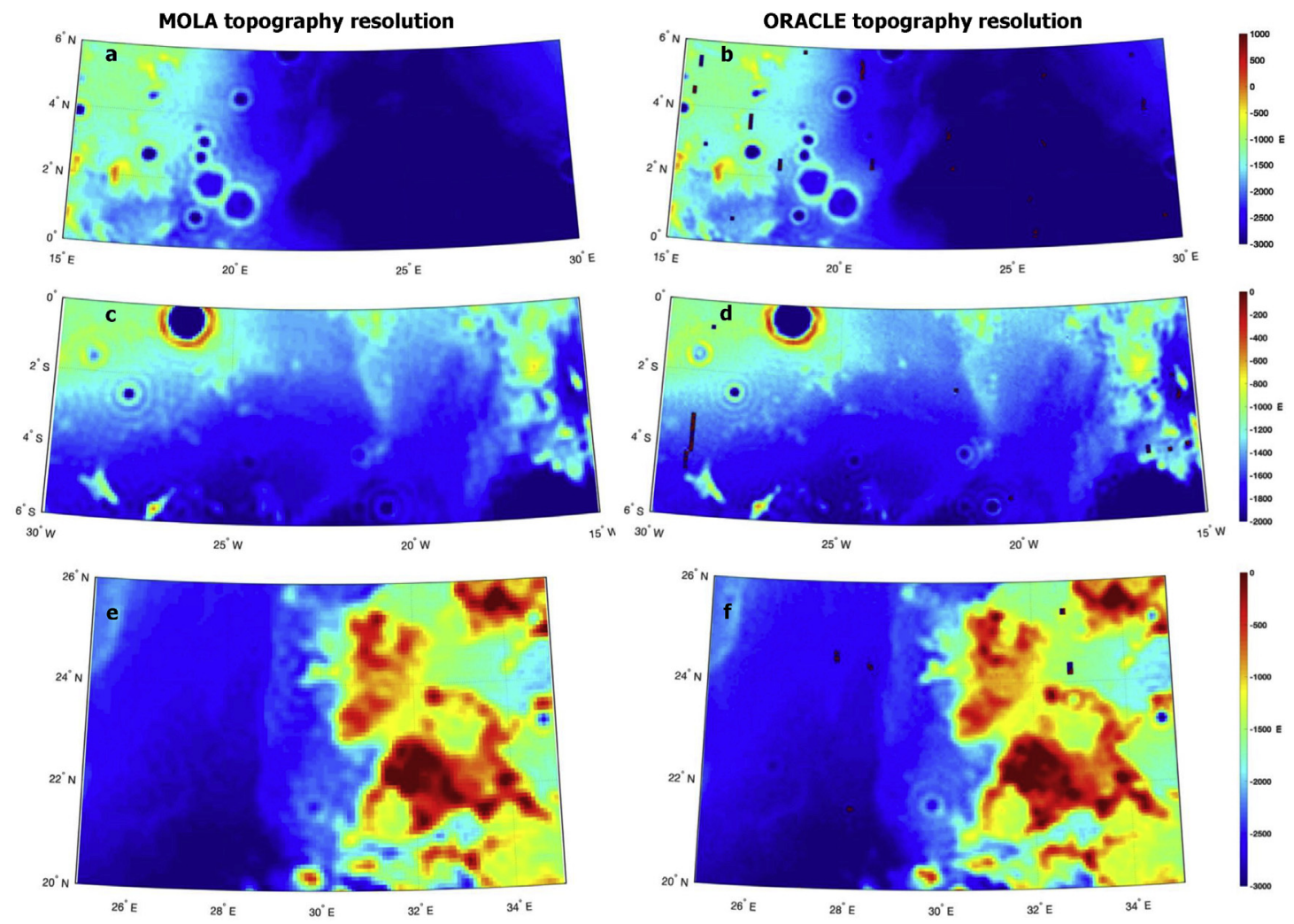

Fig. 9. Local topographic maps over regions of the lunar landing sites of the Apollo 11 (a-b), 12 and 14 (c-d), and 17 (e-f) missions reported on the Martian surface. Panels on the left and right represent MOLA and Mars ORACLE topographic maps, respectively. The Mars ORACLE laser altimeter enables a resolution in spherical harmonics to degree and order 1500 with few gaps due to the ground distribution. MOLA resolution is represented by expanding the Moon's topography to degree and order 1152 .

\section{Acknowledgments}

This study was supported by the Rita Levi Montalcini Programme of the Italian Ministry of Education, University and Research (MIUR). A.G. thanks A. Anselmi (Thales Alenia Space), V. Dehant (Royal Observatory of Belgium), D. Durante (Sapienza University of Rome) F. Forget (LMD, CNRS, Paris), S. Goossens (CRESST- NASA GSFC), H. Hussmann (DLR), L. Iess (Sapienza University of Rome), O. Karatekin (Royal Observatory of Belgium), M. Mastrogiuseppe (Caltech), E. Mazarico (NASA GSFC), G. Mitri (IRSPS, University d'Annunzio), O. Ruesch (ESA, Research Fellow), A. Stark (DLR), T. Van Hoolst (Royal Observatory of Belgium), and M. Wieczorek (Observatoire de la Cote d'Azur) for ideas and helpful discussions. A.G. is grateful to V. Notaro (Sapienza University of Rome) for her help with mass and power budget definition of the ORACLE mission.

\section{References}

[1] J. Vago, O. Witasse, H. Svedhem, P. Baglioni, A. Haldemann, G. Gianfiglio, T. Blancquaert, D. McCoy, R. de Groot, ESA ExoMars program: the next step in exploring Mars, Sol. Syst. Res. 49 (7) (2015) 518-528, https://doi.org/10.1134/ S0038094615070199.

[2] B.M. Jakosky, R. Lin, J. Grebowsky, J. Luhmann, D. Mitchell, G. Beutelschies, T. Priser, M. Acuna, L. Andersson, D. Baird, et al., The Mars atmosphere and volatile evolution (MAVEN) mission, Space Sci. Rev. 195 (1-4) (2015) 3-48, https://doi. org/10.1007/s11214-015-0139-x.

[3] M.P. Panning, P. Lognonné, W.B. Banerdt, R. Garcia, M. Golombek, S. Kedar, B. Knapmeyer-Endrun, A. Mocquet, N.A. Teanby, J. Tromp, et al., Planned products of the Mars structure service for the InSight mission to Mars, Space Sci. Rev. 211 (1-4) (2017) 611-650, https://doi.org/10.1007/s11214-016-0317-5.

[4] F. Forget, F. Hourdin, O. Talagrand, $\mathrm{CO}_{2}$ snowfall on Mars: simulation with a general circulation model, Icarus 131 (2) (1998) 302-316, https://doi.org/10. 1006/icar.1997.5874.

[5] D.E. Smith, M.T. Zuber, G.A. Neumann, Seasonal variations of snow depth on Mars, Science 294 (5549) (2001) 2141-2146, https://doi.org/10.1126/science.1066556.

[6] M. Litvak, I. Mitrofanov, A. Kozyrev, A. Sanin, V. Tret'yakov, W. Boynton, C. Shinohara, D. Hamara, S. Saunders, D. Drake, Seasonal carbon dioxide depositions on the Martian surface as revealed from neutron measurements by the HEND instrument onboard the 2001 Mars Odyssey spacecraft, Sol. Syst. Res. 38 (3) (2004) 167-177.

[7] M. Wolff, Direct measurements of the earth's gravitational potential using a satellite pair, J. Geophys. Res. 74 (22) (1969) 5295-5300, https://doi.org/10.1029/ JB074i022p05295.

[8] B.D. Tapley, S. Bettadpur, M. Watkins, C. Reigber, The gravity recovery and climate experiment: mission overview and early results, Geophys. Res. Lett. 31 (9) (2004) L09607, https://doi.org/10.1029/2004GL019920.

[9] M.T. Zuber, D.E. Smith, D.H. Lehman, T.L. Hoffman, S.W. Asmar, M.M. Watkins, Gravity recovery and interior laboratory (GRAIL): mapping the lunar interior from crust to core, GRAIL: Mapping the Moon's Interior, Springer, 2012, pp. 3-24.

[10] G.A. Neumann, D.E. Smith, M.T. Zuber, Two Mars years of clouds detected by the Mars orbiter laser altimeter, J. Geophys. Res.: Planets 108 (E4) (2003) 5023, https://doi.org/10.1029/2002JE001849.

[11] R.M. Haberle, F. Forget, A. Colaprete, J. Schaeffer, W.V. Boynton, N.J. Kelly, M.A. Chamberlain, The effect of ground ice on the Martian seasonal CO2 cycle, Planet. Space Sci. 56 (2) (2008) 251-255, https://doi.org/10.1016/j.pss.2007.08. 006.

[12] R.M. Haberle, J.B. Pollack, J.R. Barnes, R.W. Zurek, C.B. Leovy, J.R. Murphy, H. Lee, J. Schaeffer, Mars atmospheric dynamics as simulated by the NASA Ames General Circulation Model: 1 . The zonal-mean circulation, J. Geophys. Res.: Planets 98 (E2) (1993) 3093-3123, https://doi.org/10.1029/92JE02946.

[13] F. Forget, F. Hourdin, R. Fournier, C. Hourdin, O. Talagrand, M. Collins, S.R. Lewis, P.L. Read, J.-P. Huot, Improved general circulation models of the Martian atmosphere from the surface to above 80 km, J. Geophys. Res.: Planets 104 (E10) (1999) 24155-24175, https://doi.org/10.1029/1999JE001025.

[14] L. Neary, F. Daerden, The GEM-Mars general circulation model for Mars: description and evaluation, Icarus 300 (2018) 458-476, https://doi.org/10.1016/j.icarus. 2017.09.028.

[15] M.L. Litvak, I. Mitrofanov, A. Kozyrev, A. Sanin, V. Tretyakov, W.V. Boynton, N. Kelly, D. Hamara, C. Shinohara, R. Saunders, Comparison between polar regions of Mars from HEND/Odyssey data, Icarus 180 (1) (2006) 23-37, https://doi.org/ 10.1016/j icarus.2005.08.009.

[16] M.T. Zuber, F.G. Lemoine, D.E. Smith, A.S. Konopliv, S.E. Smrekar, S.W. Asmar, Mars Reconnaissance Orbiter radio science gravity investigation, J. Geophys. Res.: Planets 112 (E5) (2007) E05S07, https://doi.org/10.1029/2006JE002833.

[17] E. Van den Acker, T. Van Hoolst, O. de Viron, P. Defraigne, F. Forget, F. Hourdin, $\mathrm{V}$. Dehant, Influence of the seasonal winds and the $\mathrm{CO} 2$ mass exchange between atmosphere and polar caps on Mars' rotation, J. Geophys. Res.: Planets 107 (E7) (2002), https://doi.org/10.1029/2000JE001539 9-1.

[18] Ö. Karatekin, O. de Viron, S. Lambert, V. Dehant, P. Rosenblatt, T. Van Hoolst, S. Le Maistre, Atmospheric angular momentum variations of Earth, Mars and Venus at 
seasonal time scales, Planet. Space Sci. 59 (10) (2011) 923-933, https://doi.org/10. 1016/j.pss.2010.09.010.

[19] A.S. Konopliv, S.W. Asmar, W.M. Folkner, Ö. Karatekin, D.C. Nunes, S.E. Smrekar, C.F. Yoder, M.T. Zuber, Mars high resolution gravity fields from MRO, Mars seasonal gravity, and other dynamical parameters, Icarus 211 (1) (2011) 401-428, https://doi.org/10.1016/j.icarus.2010.10.004.

[20] A. Genova, S. Goossens, F.G. Lemoine, E. Mazarico, G.A. Neumann, D.E. Smith, M.T. Zuber, Seasonal and static gravity field of Mars from MGS, Mars Odyssey and MRO radio science, Icarus 272 (2016) 228-245, https://doi.org/10.1016/j.icarus. 2016.02.050.

[21] I. Mitrofanov, M. Zuber, M. Litvak, W.V. Boynton, D. Smith, D. Drake, D. Hamara, A. Kozyrev, A. Sanin, C. Shinohara, et al., $\mathrm{CO}_{2}$ snow depth and subsurface water-ice abundance in the northern hemisphere of Mars, Science 300 (5628) (2003) 2081-2084, https://doi.org/10.1126/science.1084350.

[22] S. Piqueux, A. Kleinböhl, P.O. Hayne, D.M. Kass, J.T. Schofield, D.J. McCleese, Variability of the Martian seasonal $\mathrm{CO}_{2}$ cap extent over eight Mars Years, Icarus 251 (2015) 164-180, https://doi.org/10.1016/j.icarus.2014.10.045.

[23] D.E. Smith, M.T. Zuber, H.V. Frey, J.B. Garvin, J.W. Head, D.O. Muhleman, G.H. Pettengill, R.J. Phillips, S.C. Solomon, H.J. Zwally, et al., Mars orbiter laser altimeter: experiment summary after the first year of global mapping of Mars, J. Geophys. Res.: Planets 106 (E10) (2001) 23689-23722, https://doi.org/10.1029/ 2000JE001364.

[24] M.T. Zuber, D. Smith, S. Solomon, D. Muhleman, J. Head, J. Garvin, J. Abshire, J. Bufton, The Mars Observer laser altimeter investigation, J. Geophys. Res.: Planets 97 (E5) (1992) 7781-7797, https://doi.org/10.1029/92JE00341.

[25] J.B. Abshire, X. Sun, R.S. Afzal, Mars Orbiter Laser Altimeter: receiver model and performance analysis, Appl. Optic. 39 (15) (2000) 2449-2460, https://doi.org/10. 1364/AO.39.002449.

[26] A.S. Konopliv, R.S. Park, W.M. Folkner, An improved JPL Mars gravity field and orientation from Mars orbiter and lander tracking data, Icarus 274 (2016) 253-260, https://doi.org/10.1016/j.icarus.2016.02.052.

[27] M.A. Wieczorek, G.A. Neumann, F. Nimmo, W.S. Kiefer, G.J. Taylor, H.J. Melosh, R.J. Phillips, S.C. Solomon, J.C. Andrews-Hanna, S.W. Asmar, et al., The crust of the Moon as seen by GRAIL, Science 339 (6120) (2013) 671-675, https://doi.org/10. 1126/science.1231530.

[28] S. Goossens, T.J. Sabaka, A. Genova, E. Mazarico, J.B. Nicholas, G.A. Neumann, Evidence for a low bulk crustal density for Mars from gravity and topography, Geophys. Res. Lett. 44 (15) (2017) 7686-7694, https://doi.org/10.1002/ 2017GL074172.

[29] M.A. Wieczorek, Constraints on the composition of the Martian south polar cap from gravity and topography, Icarus 196 (2) (2008) 506-517, https://doi.org/10 1016/j.icarus.2007.10.026

[30] V. Dehant, S. Le Maistre, A. Rivoldini, M. Yseboodt, P. Rosenblatt, T. Van Hoolst, M. Mitrovic, Ö. Karatekin, J. Marty, A. Chicarro, Revealing Mars' deep interior: future geodesy missions using radio links between landers, orbiters, and the Earth, Planet. Space Sci. 59 (10) (2011) 1069-1081, https://doi.org/10.1016/j.pss.2010. 03.014.

[31] S. Le Maistre, P. Rosenblatt, A. Rivoldini, V. Dehant, J.-C. Marty, O. Karatekin, Lander radio science experiment with a direct link between Mars and the Earth, Planet. Space Sci. 68 (1) (2012) 105-122, https://doi.org/10.1016/j.pss.2011.12 020 .

[32] G.A. Rauwolf, V.L. Coverstone-Carroll, Near-optimal low-thrust orbit transfers generated by a genetic algorithm, J. Spacecr. Rocket. 33 (6) (1996) 859-862, https://doi.org/10.2514/3.26850.

[33] J. Benkhoff, J. Van Casteren, H. Hayakawa, M. Fujimoto, H. Laakso, M. Novara, P. Ferri, H.R. Middleton, R. Ziethe, BepiColombo - comprehensive exploration of Mercury: mission overview and science goals, Planet. Space Sci. 58 (1-2) (2010) 2-20, https://doi.org/10.1016/j.pss.2009.09.020.

[34] J. Snyder, D.M. Goebel, R.R. Hofer, J.E. Polk, N.C. Wallace, H. Simpson, Performance evaluation of the T6 ion engine, J. Propuls. Power 28 (2) (2012) 371-379, https://doi.org/10.2514/1.B34173.

[35] M. Vasile, L. Summerer, P. De Pascale, Design of Earth-Mars transfer trajectories using evolutionary-branching technique, Acta Astronaut. 56 (8) (2005) 705-720, https://doi.org/10.1016/j.actaastro.2004.12.002.

[36] D.A. Spencer, R. Tolson, Aerobraking cost and risk decisions, J. Spacecr. Rocket. 44 (6) (2007) 1285-1293, https://doi.org/10.2514/1.24303.

[37] R.W. Zurek, S.E. Smrekar, An overview of the Mars Reconnaissance Orbiter (MRO) science mission, J. Geophys. Res.: Planets 112 (E5) (2007) E05S01, https://doi.org/ 10.1029/2006JE002701.

[38] R. Floberghagen, The Far Side, Lunar Gravimetry in the New Millennium, Ph.D. thesis (2001) ISBN 90-9014693-8, TU Delft.

[39] J. Ouaknine, L. Blarre, L. Oddos-Marcel, J. Montel, J.-M. Julio, Reduction of low frequency error for SED36 and APS based HYDRA star trackers, International Conference on Space Optics-ICSO, vol. 10567, International Society for Optics and Photonics, 2006, p. 105672R, https://doi.org/10.1117/12.2308086 2017.

[40] R. Tolson, G. Keating, G. Cancro, J. Parker, S. Noll, B. Wilkerson, Application of accelerometer data to Mars Global Surveyor aerobraking operations, J. Spacecr. Rocket. 36 (3) (1999) 323-329, https://doi.org/10.2514/2.3474.

[41] Press Kit, Mars Reconnaissance Orbiter Arrival, Tech. Rep. National Aeronautics and Space Administration, 2006.

[42] M. Arena, P.V. Giove, D. Di Giuliomaria, A. Gizzi, S. Ciarcia, C.O. Simone, M.G. Campagna, D. Gelfusa, Next generation transponders for interplanetary mission: integrated radioscience and deep space TT\&C transponder, 7th ESA International Workshop on Tracking, Telemetry and Command Systems for Space Applications, ESTEC, Noordwijk, The Netherlands, 2016.

[43] D.K. Srinivasan, M.E. Perry, K.B. Fielhauer, D.E. Smith, M.T. Zuber, The radio frequency subsystem and radio science on the MESSENGER mission, Space Sci. Rev. 131 (1-4) (2007) 557-571, https://doi.org/10.1007/s11214-007-9270-7.
[44] S. Asmar, D. Atkinson, M. Bird, G. Wood, Ultra-stable oscillators for planetary entry probes, Planet. Probe Atmos. Entry Descent Trajectory Anal. Sci. 544 (2004) $131-134$.

[45] C. Dunn, W. Bertiger, Y. Bar-Sever, S. Desai, B. Haines, D. Kuang, G. Franklin, I. Harris, G. Kruizinga, T. Meehan, Instrument of GRACE GPS augments gravity measurements, GPS World 14 (2) (2003) 16-29.

[46] W.M. Klipstein, B.W. Arnold, D.G. Enzer, A.A. Ruiz, J.Y. Tien, R.T. Wang, C.E. Dunn, The lunar gravity ranging system for the gravity recovery and interior laboratory (grail) mission, GRAIL: Mapping the Moon's Interior, Springer, 2013, pp. 57-76.

[47] S. Asmar, J. Armstrong, L. Iess, P. Tortora, Spacecraft Doppler tracking: noise budget and accuracy achievable in precision radio science observations, Radio Sci. 40 (2) (2005) RS2001, https://doi.org/10.1029/2004RS003101.

[48] S. Ciarcia, L. Simone, D. Gelfusa, P. Colucci, G. De Angelis, F. Argentieri, L. Iess, R. Formaro, MORE and Juno Ka-band transponder design, performance, qualification and in-flight validation, Image 15 (2013) F1.

[49] L. Iess, G. Boscagli, Advanced radio science instrumentation for the mission BepiColombo to Mercury, Planet. Space Sci. 49 (14-15) (2001) 1597-1608, https:// doi.org/10.1016/S0032-0633(01)00096-4.

[50] D. Titov, S. Barabash, L. Bruzzone, M. Dougherty, C. Erd, L. Fletcher, P. Gare, R. Gladstone, O. Grasset, L. Gurvits, et al., JUICE: complementarity of the payload in addressing the mission science objectives, EGU General Assembly Conference Abstracts, vol. 16, 2014

[51] Space Frequency Coordination Group, Frequency Assignment Guidelines for Communications in the Mars Region, Recommendation SFCG 22-1R2, Tech. Rep. Consultative Committee for Space Data System, 13 September 2017.

[52] T. Okada, T. Fukuhara, S. Tanaka, M. Taguchi, T. Imamura, T. Arai, H. Senshu, Y. Ogawa, H. Demura, K. Kitazato, et al., Thermal infrared imaging experiments of C-type asteroid 162173 Ryugu on Hayabusa2, Space Sci. Rev. 208 (1-4) (2017) 255-286, https://doi.org/10.1007/s11214-016-0286-8.

[53] D.E. Smith, M.T. Zuber, G.B. Jackson, J.F. Cavanaugh, G.A. Neumann, H. Riris, X. Sun, R.S. Zellar, C. Coltharp, J. Connelly, et al., The lunar orbiter laser altimeter investigation on the lunar reconnaissance orbiter mission, Space Sci. Rev. 150 (1-4) (2010) 209-241, https://doi.org/10.1007/s11214-009-9512-y.

[54] N. Thomas, T. Spohn, J.-P. Barriot, W. Benz, G. Beutler, U. Christensen, V. Dehant, C. Fallnich, D. Giardini, O. Groussin, et al., The BepiColombo Laser Altimeter (BELA): concept and baseline design, Planet. Space Sci. 55 (10) (2007) 1398-1413, https://doi.org/10.1016/j.pss.2007.03.003.

[55] X. Sun, F.M. Davidson, L. Boutsikaris, J.B. Abshire, Receiver characteristics of laser altimeters with avalanche photodiodes, IEEE Trans. Aerosp. Electron. Syst. 28 (1) (1992) 268-275, https://doi.org/10.1109/7.135452.

[56] J.F. Cavanaugh, J.C. Smith, X. Sun, A.E. Bartels, L. Ramos-Izquierdo, D.J. Krebs, J.F. McGarry, R. Trunzo, A.M. Novo-Gradac, J.L. Britt, et al., The Mercury laser altimeter instrument for the MESSENGER mission, Space Sci. Rev. 131 (1-4) (2007) 451-479, https://doi.org/10.1007/s11214-007-9273-4.

[57] W.M. Kaula, Theory of Satellite Geodesy: Applications of Satellites to Geodesy, Courier Corporation, Mineola, NY, 2013.

[58] D. Smith, M. Zuber, H. Frey, J. Garvin, J. Head, D. Muhleman, G. Pettengill, R. Phillips, S. Solomon, H. Zwally, et al., Topography of the northern hemisphere of Mars from the Mars orbiter laser altimeter, Science 279 (5357) (1998) 1686-1692, https://doi.org/10.1126/science.279.5357.1686.

[59] D.E. Smith, M.T. Zuber, G.A. Neumann, F.G. Lemoine, E. Mazarico, M.H. Torrence, J.F. McGarry, D.D. Rowlands, J.W. Head, T.H. Duxbury, et al., Initial observations from the lunar orbiter laser altimeter (LOLA), Geophys. Res. Lett. 37 (18) (2010) L18204, https://doi.org/10.1029/2010GL043751.

[60] R. Seu, D. Biccari, R. Orosei, L. Lorenzoni, R. Phillips, L. Marinangeli, G. Picardi, A. Masdea, E. Zampolini, SHARAD: the MRO 2005 shallow radar, Planet. Space Sci. 52 (1-3) (2004) 157-166, https://doi.org/10.1016/j.pss.2003.08.024.

[61] J. Klokocnik, A. Bezdek, J. Kostelecky, J. Sebera, Orbit tuning of planetary orbiters for accuracy gain in gravity field mapping, J. Guid. Control Dyn. 33 (3) (2010) 853-861, https://doi.org/10.2514/1.46223.

[62] S. Bruinsma, F.G. Lemoine, A preliminary semiempirical thermosphere model of Mars: DTM-Mars, J. Geophys. Res.: Planets 107 (E10) (2002), https://doi.org/10. 1029/2001JE001508 15-1.

[63] R. Park, S.W. Asmar, E.G. Fahnestock, A.S. Konopliv, W. Lu, M.M. Watkins, Gravity recovery and interior laboratory simulations of static and temporal gravity field, J. Spacecr. Rocket. 49 (2) (2012) 390-400, https://doi.org/10.2514/1.A32117.

[64] P. Touboul, E. Willemenot, B. Foulon, V. Josselin, Accelerometers for champ, grace and goce space missions: synergy and evolution, Boll. Geofis. Teor. Appl. 40 (3-4) (1999) 321-327.

[65] D.M. Lucchesi, V. Iafolla, The non-gravitational perturbations impact on the bepicolombo radio science experiment and the key role of the isa accelerometer: direct solar radiation and albedo effects, Celest. Mech. Dyn. Astron. 96 (2) (2006) 99-127, https://doi.org/10.1007/s10569-006-9034-9.

[66] S.B. Luthcke, H. Zwally, W. Abdalati, D. Rowlands, R. Ray, R. Nerem, F. Lemoine, J. McCarthy, D. Chinn, Recent Greenland ice mass loss by drainage system from satellite gravity observations, Science 314 (5803) (2006) 1286-1289, https://doi. org/10.1126/science.1130776.

[67] G.A. Neumann, D.D. Rowlands, F.G. Lemoine, D.E. Smith, M.T. Zuber, Crossover analysis of Mars orbiter laser altimeter data, J. Geophys. Res.: Planets 106 (E10) (2001) 23753-23768, https://doi.org/10.1029/2000JE001381.

[68] H. Save, S. Bettadpur, B.D. Tapley, High-resolution CSR GRACE RL05 mascons, J. Geophys. Res.: Solid Earth 121 (10) (2016) 7547-7569, https://doi.org/10.1002/ 2016JB013007.

[69] R. Orosei, S.E. Lauro, E. Pettinelli, A. Cicchetti, M. Coradini, B. Cosciotti, F. Di Paolo, E. Flamini, E. Mattei, M. Pajola, et al., Radar evidence of subglacial liquid water on Mars, Science 361 (6401) (2018) 490-493, https://doi.org/10.1126/ science.aar7268. 